weite Teile der Gesellschaft in das System inkludiert, wodurch eine Grundlage für die Akzeptanz herbeigeführter Entscheidungen entstehen kann. ${ }^{37}$

Angesichts des Defizits an Inklusionsmechanismen ist zu bezweifeln, dass in Liquid Democracy ein Ersatz für bestehende repräsentative Strukturen gesehen werden kann. Im Rahmen einer Diskussion um die mögliche Ergänzung des etablierten Systems durch Liquid Democracy-Elemente muss zwangsläufig gefragt werden, wie das Kriterium substantieller politischer Gleichheit gewahrt werden kann. Die Notwendigkeit, sich dieser Debatte zu stellen, haben die politischen Akteure erkannt. So hat der Deutsche Bundestag die Enquete-Kommission „Internet und digitale Gesellschaft " einberufen ${ }^{38}$, welche die Zukunft der digitalen Gesellschaft zum Thema hat und dabei Bürger mittels Adhocracy in die Diskussion einbinden will. ${ }^{39}$

37 Vgl. Enquete-Kommission Internet und Digitale Gesellschaft, Wer treibt den Strukturwandel der politischen Kommunikation und Partizipation voran - und wo bleiben die Anderen?, https://www. bundestag.de/internetenquete/dokumentation/Sitzungen/20120319/A-Drs_17_24_049-B_-_ Stellungnahme_Prof_Dr_Vowe_19_3_2012.pdf (Abruf am 2. Juli 2012), S. 6 f. Die Defizite repräsentativer Arrangements in unterschiedlichen Dimensionen sollen durch diesen Verweis nicht abgeschwächt oder ausgeblendet werden.

38 Enquete-Kommission Internet und Digitale Gesellschaft, Wissen, Ideen und Expertise für die Politik, https://enquetebeteiligung.de/ (Abruf am 2. Juli 2012).

39 Für hilfreiche Hinweise und Kritik einer früheren Fassung des Manuskripts danke ich Prof. Walter Reese-Schäfer und den Teilnehmern seines Doktorandenkolloquiums.

\title{
Parlamentarisches Ordnungsgeld und Sitzungsausschluss: Verfassungsrechtliche Aspekte ${ }^{*}$
}

\author{
Oliver Borowy
}

Der Deutsche Bundestag hat in seiner Sitzung am 30. Juni 2011 beschlossen, sein parlamentarisches Ordnungsrecht um eine weitere Ordnungsmaßnahme, das so genannte Ordnungsgeld, zu ergänzen. ${ }^{1}$ Der sitzungsleitende Präsident ${ }^{2}$ kann nunmehr bei einer nicht nur geringfügigen Verletzung der Ordnung oder der Würde des Bundestages ein Ordnungsgeld in Höhe von 1.000 Euro, im Wiederholungsfall von 2.000 Euro festsetzen. Mit dem Ordnungsgeld soll nach dem Willen des Parlaments eine im Sanktionssystem der Geschäftsordnung vorhandene Lücke geschlossen werden, die zwischen dem Ordnungsruf am unteren Ende und dem Sitzungsausschluss am oberen Ende der Skala besteht, um dem Präsidenten

* Zugleich eine Erwiderung auf Albert Ingold / Sophie-Charlotte Lenski, Ordnungsgeld und Sitzungsausschluss als Ordnungsmaßnahmen gegen Bundestagsabgeordnete, in: JZ, 67. Jg. (2012), H. 3, S. 120 - 125. Der Verfasser ist Mitarbeiter der Bundestagsverwaltung. Der Beitrag gibt allein seine persönliche Auffassung wieder.

1 BT-Plenarprotokoll 17/117 vom 30. Juni 2011, S. 13531 C.

2 Im weiteren Text wird nur vom Präsidenten gesprochen. Gemeint ist damit die jeweils sitzungsleitende Präsidentin beziehungsweise der jeweils sitzungsleitende Präsident, sofern nicht ausdrücklich vom Bundestagspräsidenten die Rede ist. 
so ein im Einzelfall angemessenes Einschreiten zu ermöglichen. ${ }^{3}$ Denn der Ordnungsruf ( $\$ 36$ Abs. 1 GO-BT) bleibe letztlich ohne einschneidende Folgen für den betroffenen Abgeordneten, während der Sitzungsausschluss (\$ 38 GO-BT) wegen des mit ihm verbundenen Entzugs des Rede- und Stimmrechts als schärfstes Ordnungsmittel häufig unangemessen erscheine. Anlass für die Einführung des Ordnungsgeldes waren vor allem wiederholte „demonstrationsähnliche Störungen“ im Plenum während der vorangegangenen und laufenden Wahlperiode. ${ }^{4}$ Mit den Vorgängen hatte sich auch der Ältestenrat befasst und ausdrücklich die Auffassung des Präsidenten geteilt, dass derartige Störungen im Plenarsaal als gröbliche Ordnungsverletzung anzusehen seien. ${ }^{5}$

Im Zuge der Einführung des Ordnungsgeldes wurden die bisherigen $\$ \$ 36 \mathrm{ff}$. der Geschäftsordnung des Deutschen Bundestages (GO-BT) neu gefasst und das Abgeordnetengesetz $(\mathrm{AbgG})$ durch Einfügung eines neuen $\$ 44 \mathrm{a}$ Abs. 5 ergänzt. Sowohl die Ergänzung des Abgeordnetengesetzes als auch die Geschäftsordnungsänderung traten wegen der zwischen den Regelungen bestehenden inhaltlichen Verknüpfung gleichzeitig am 8. Dezember 2011 in Kraft. ${ }^{6}$ Die Neuregelungen waren nicht nur im politischen Raum umstritten ${ }^{7}$, sondern stießen auch kurz nach ihrer Einführung im Schrifttum ${ }^{8}$ auf verfassungsrechtliche Bedenken. Im Folgenden soll deshalb unter besonderer Berücksichtigung der parlamentarischen Praxis, insbesondere der Abläufe im Plenum des Bundestages, auf die Kritik näher eingegangen werden.

\section{Rechtsgrundlagen, Zweck und verfassungsrechtliche Grenzen der parlamentarischen Ordnungsgewalt}

\subsection{Rechtsgrundlagen der Ordnungsgewalt}

Gegenüber den Abgeordneten des Bundestages ist die Ordnungs- oder Disziplinargewalt Ausfluss der dem Parlament durch Artikel 40 Abs. 1 Satz 2 GG eingeräumten Geschäftsordnungsautonomie, die das Parlament zur Aufstellung von Regeln berechtigt, die ein ord-

3 BT-Drucksache 17/5471 vom 12. April 2011, S. 3; BT-Drucksache 17/6309 vom 29. Juni 2011, S. 5.

4 Vgl. zum Beispiel BT-Plenarprotokoll 16/25 vom 16. März 2006, S. 1919 B (Anziehen von Streikwesten mit Aufdruck); BT-Plenarprotokoll 16/135 vom 16. Januar 2008, S. 14242 D (Aufsetzen von Masken mit dem Abbild eines Politikers mit Pinocchio-Nase); BT-Plenarprotokoll 17/25 vom 26. Februar 2010, S. 2168 D (Hochhalten von Spruchbändern mit Namen der Opfer des Kunduz-Vorfalls); BT-Plenarprotokoll 17/60 vom 17. September 2010, S. 6285 B (Tragen von T-Shirts mit Stuttgart 21-Aufdruck).

5 Vgl. Heinrich G. Ritzel/ Joseph Bücker / Hermann J. Schreiner, Handbuch für die Parlamentarische Praxis II, Loseblatt: Stand: 28. Lfg. 12/2011, \$38, Anmerkung 2 a).

6 Änderungen der Geschäftsordnung des Bundestages treten ansonsten grundsätzlich bereits mit Plenarbeschluss in Kraft (Gerald Kretschmer, Geschäftsordnungen deutscher Volksvertretungen, in: Hans-Peter Schneider / Wolfgang Zeh (Hrsg.), Parlamentsrecht und Parlamentspraxis, Berlin / New York 1989, \$9 Rz. 128.

7 Die Änderungen des Abgeordnetengesetzes und der Geschäftsordnung wurden mit den Stimmen der Fraktionen der CDU/CSU, SPD und FDP gegen die Stimmen der Fraktionen Die Linke und Bündnis 90/Die Grünen beschlossen; vgl. BT- Plenarprotokoll 17/117 vom 30. Juni 2011, S. 13531 C.

8 Vgl. Albert Ingold / Sophie-Charlotte Lenski, Ordnungsgeld und Sitzungsausschluss als Ordnungsmaßnahmen gegen Bundestagsabgeordnete, in: JZ, 67. Jg. (2012), H. 3, S. 120 - 125. 
nungsgemäßes sowie dem Ansehen und der Würde des Hauses entsprechendes Arbeiten gewährleisten. ${ }^{9}$ Die Geschäftsordnungsautonomie erstreckt sich dabei traditionell auf die Bereiche „Geschäftsgang“ und „Disziplin“. ${ }^{10}$ Originärer Träger beziehungsweise Inhaber der Ordnungsgewalt ist der Bundestag in der Gesamtheit seiner Mitglieder, was auch in $\$ 39$ GO-BT zum Ausdruck kommt, wonach das Plenum und nicht der Präsident über Einsprüche gegen Ordnungsmaßnahmen entscheidet. Der Bundestag hat die Ausübung seiner Ordnungsgewalt auf den sitzungsleitenden Präsidenten übertragen ( $\$ 7$ Abs. 1 Satz 2 GO-BT), der sie in den Plenarsitzungen des Bundestages nach Maßgabe der Ordnungsbestimmungen der $\$ \$ 36$ bis 41 GO-BT ,in eigener Verantwortung und unabhängig “ ausübt. ${ }^{11}$ Dadurch sollen, wie bei der Sitzungsleitung insgesamt, vor allem Zufallsentscheidungen je nach politischen Mehrheitsverhältnissen vermieden und so insgesamt eine gerechte und kontinuierliche Handhabung der Ordnungsgewalt gewährleistet werden. ${ }^{12}$

\subsection{Inhalt und Zweck der Ordnungsgewalt}

Eine Legaldefinition der parlamentarischen Ordnung enthält weder das Grundgesetz noch die Geschäftsordnung des Bundestages; es handelt sich vielmehr um einen unbestimmten Rechtsbegriff. ${ }^{13}$ Die herrschende Meinung in Rechtsprechung und Schrifttum versteht unter der parlamentarischen Ordnung „die Gesamtheit der Normen, deren Befolgung nach den im Parlament herrschenden - nur selten und ungern wechselnden - Anschauungen als Vorbedingung einer gedeihlichen, das Staatsleben fördernden Beratung der Abgeordneten und als Grundlage des innerparlamentarischen Lebens gilt"14. Der Begriff erstreckt sich dabei auch auf geschriebene und ungeschriebene Regeln des Parlamentsrechts und des Parlamentsbrauchs. ${ }^{15}$ So widerspricht es beispielsweise überkommenem Parlamentsbrauch,

9 BVerfGE 44, S. 308, S. 315; BVerfGE 60, S. 374, S. 379; BVerfGE 80, S. 188, S. 218 f.; Michael Köhler, Die Rechtsstellung des Parlamentspräsidenten in den Ländern der Bundesrepublik Deutschland und ihre Aufgaben im parlamentarischen Geschäftsgang, Berlin 2000, S. 180; Joseph Bücker, Das parlamentarische Ordnungsrecht, in: Hans-Peter Schneider / Wolfgang Zeh, a.a.O. (Fn. 6), $\$ 34$ Rz. 3; Heinrich G. Ritzel / Joseph Bücker / Hermann J. Schreiner, a.a.O. (Fn. 5), Vorbemerkung zu $\$ \$ 36-41$, Anmerkung 4 a), dort unter Anmerkung 2 c) auch zur dogmatischen Begründung der gegenüber Sitzungsteilnehmern, die nicht Mitglieder des Parlaments sind, und Zuhörern bestehenden Ordnungsgewalt; vgl. auch LVerfG Mecklenburg-Vorpommern, in: NordÖR 2009, S. 205, S. 206; anderer Auffassung Albert Ingold / Sophie-Charlotte Lenski, a.a.O. (Fn. 8), S. 120, S. 122.

10 BVerfGE 44, S. 308, S. 315.

11 BVerfGE 60, S. 374, S. 379; Joseph Bücker, a.a.O. (Fn. 6), \$34 Rz. 3; Hans Trossmann (Hrsg.), Parlamentsrecht des Deutschen Bundestages, München 1977, $\$ 7$ Rz. 34; Lars Brocker, in: Rudolf Dolzer / Karin Graßhof / Wolfgang Kahl / Christian Waldhoff(Hrsg.), Bonner Kommentar zum Grundgesetz, Heidelberg 2012, Art. 40 Rz. 134 (Drittbearbeitung Februar 2011).

12 Vgl. Joseph Bücker, a.a.O. (Fn. 6), \$34 Rz. 3; Lars Brocker, a.a.O. (Fn. 11), Art. 40 Rz. 132.

13 Vgl. Joseph Bücker, a.a.O. (Fn. 6), \$34 Rz. 15; Michael Köhler, a.a.O. (Fn. 9), S. 175; vgl. auch LVerfG Mecklenburg-Vorpommern, Urteil vom 27. Januar 2011 - 04/09, juris Rz. 28.

14 So bereits Hermann F. Schmid, Parlamentarische Disziplin. Eine rechtsvergleichende Studie, in: AöR Bd. 32 (1914), S. 439 - 579, S. 439, S. 498; vgl. auch Lars Brocker, a.a.O. (Fn. 11), Art. 40 Rz. 131; Michael Köhler, a.a.O. (Fn. 9), S. 175; vgl. auch LVerfG Mecklenburg-Vorpommern, in: NordÖR 2009, S. 205, S. 207.

15 Vgl. Joseph Bücker, a.a.O. (Fn. 6), $\$ 34$ Rz. 7; vgl. auch VerfGH Sachsen, in: NVwZ-RR 2012, S. 89, S. 90. 
Ansehen und Autorität des Präsidenten durch in der Plenarsitzung geäußerte Kritik an seiner Verhandlungsführung zu beeinträchtigen. ${ }^{16}$ Auch wird zu Recht die Erhaltung von Würde und Ansehen des Parlaments als Schutzgut der Ordnungsgewalt angesehen. ${ }^{17}$

Nach Auffassung von Albert Ingold und Sophie-Charlotte Lenski ${ }^{18}$ soll der Zweck der Ordnungsgewalt sich hingegen darin erschöpfen, einen geordneten äußeren Ablauf der Plenarsitzungen zu gewährleisten, mit der Folge, dass nur Störungen unterbunden werden können, die das Plenum, insbesondere die übrigen Mitglieder des Bundestages daran hindern, ihren parlamentarischen Aufgaben ungehindert nachzukommen. Geboten sei deshalb eine restriktive Auslegung des Begriffs der parlamentarischen Ordnung durch eine Beschränkung auf die Sicherung des Sitzungsverlaufs.

Dem ist entgegenzuhalten, dass eine derartige Zweckbindung der Ordnungsgewalt im Widerspruch zu dem weiten Begriffsverständnis der Praxis steht. ${ }^{19}$ Vom amtierenden Präsidenten wird vor allem von den im Saal anwesenden Abgeordneten nicht nur erwartet, dass er für einen ungestörten „technischen“ Fortgang der Verhandlungen sorgt. An ihn richtet sich auch die berechtigte Erwartung, dass der Sitzungsverlauf insgesamt dem Ansehen des Bundestages in der Öffentlichkeit gerecht wird, denn gerade für das Parlament, das zur Wahrnehmung seiner repräsentativen Aufgaben berufen ist, ist es wichtig, insbesondere bei der Wählerschaft Akzeptanz zu finden. ${ }^{20}$ Dazu gehört, dass der Präsident auf die Einhaltung allgemein anerkannter parlamentarischer Gepflogenheiten achtet und diese auch durchsetzt, mögen sie auch auf ungeschriebenen Regeln und Parlamentsbrauch beruhen. Es sind mithin sämtliche Werte und Verhaltensweisen zu berücksichtigen, die sich in der demokratischen und vom Repräsentationsgedanken getragenen parlamentarischen Praxis entwickelt haben und die durch historische und politische Entwicklungen geformt sind. ${ }^{21}$ So geht der Bundestag fast einmütig davon aus, dass die Auseinandersetzung im Plenum nur durch Wortbeiträge erfolgt, nicht aber mittels plakativer oder stiller Protestaktionen, mit denen in erster Linie ein bestimmtes politisches Anliegen öffentlichkeitswirksam unterstrichen und eine besondere mediale Aufmerksamkeit geschaffen werden soll. Auch wenn sich derartige Aktionen nicht unmittelbar gegen den technischen Sitzungsablauf richten, müssen sie vom Prä-

16 Vgl. Heinrich G. Ritzel / Joseph Bücker / Hermann J. Schreiner, a.a.O. (Fn. 5), Vorbemerkung zu $\$ \$ 36$ - 41, Anmerkung 1 c) cc); Hans Trossmann, a.a.O. (Fn. 11), \$40 Rz. 13; vgl. auch LVerfG Mecklenburg-Vorpommern, in: NordÖR 2009, S. 205, S. 207; VerfGH Sachsen, Urteil vom 3. November 2011 - Vf. 35-I-11, juris Rz. 35.

17 Vgl. Heinrich G. Ritzel / Joseph Bücker / Hermann J. Schreiner, a.a.O. (Fn. 5), Vorbemerkung zu $\$ \$ 36$ - 41, Anmerkung 1 a); Lars Brocker, a.a.O. (Fn. 11), Art. 40 Rz. 118; Gerhard Weng, Die Würde des Hauses, in: ZParl, 17. Jg. (1986), H. 2, S. 248 - 262, S. 248, S. 255; vgl. auch LVerfG Mecklenburg-Vorpommern, in: NordÖR 2009, S. 205, S. 207; VerfGH Sachsen, in: NVwZ-RR 2011, S. 129, S. 131; anderer Auffassung Albert Ingold / Sophie-Charlotte Lenski, a.a.O. (Fn. 8), S. 120, S. 122 ff. Zur Kritik am Begriff der "Würde“ auch in den parlamentarischen Beratungen vgl. unter 3.2.

18 Vgl. Albert Ingold/ Sophie-Charlotte Lenski, a.a.O. (Fn. 8), S. 120, S. 122.

19 Vgl. Heinrich G. Ritzel/ Joseph Bücker / Hermann J. Schreiner, a.a.O. (Fn. 5), Vorbemerkung zu $\$ \$ 36$ - 41, Anmerkung 1 b) und d); vgl. auch LVerfG Mecklenburg-Vorpommern, in: NordÖR 2009, S. 205, S. 207.

20 Vgl. Heinrich G. Ritzel / Joseph Bücker / Hermann J. Schreiner, a.a.O. (Fn. 5), Vorbemerkung zu $\$ \$ 36$ - 41, Anmerkung 1 b).

21 Vgl. Michael Köhler, a.a.O. (Fn. 9), S. 167; vgl. auch LVerfG Mecklenburg-Vorpommern, in: NordÖR 2009, S. 205, S. 207. 
sidenten unterbunden werden können, schon um Widerspruch und damit verbundene Unruhe und Ablenkung bei den anwesenden Abgeordneten zu vermeiden. Ließe man solche Demonstrationen im Plenum zu, bestünde zudem die Gefahr eines Überbietungswettbewerbs um das höchste Maß an Aufmerksamkeit erregenden Darbietungsformen. ${ }^{22}$ Das parlamentarische Ordnungsrecht muss der „Prämierung“ eines größtmöglichen Klamauks aber entgegenwirken, da anderenfalls ein parlamentarisches Arbeiten unter den Bedingungen der heutigen Mediengesellschaft erheblich erschwert, wenn nicht gar ausgeschlossen wird. Auf der Grundlage eines Begriffsverständnisses, wie es von Ingold und Lenski vertreten wird, wäre dies indes nicht oder nur schwer möglich. ${ }^{23}$ Mit der nahezu einhelligen Auffassung in Rechtsprechung und Literatur ist deshalb davon auszugehen, dass das Parlament kraft seiner Geschäftsordnungsautonomie zum Erlass aller von ihm für notwendig angesehenen Regeln berechtigt ist, um ein ordnungsgemäßes und der Würde des Hauses entsprechendes Arbeiten zu gewährleisten. ${ }^{24}$

\subsection{Verfassungsrechtliche Grenzen der Ordnungsgewalt}

Sämtliche in $\$ \$ 36$ ff.-GO-BT geregelten Ordnungsmaßnahmen berühren den Abgeordneten in seinen durch Art. 38 Abs. 1 Satz 2 GG geschützten verfassungsrechtlichen Statusrechten, da der sitzungsleitende Präsident mit ihnen eine ordnungswidrige Äußerung oder ein entsprechendes Verhalten formell parlamentarisch ahndet. ${ }^{25}$ Anders als bei der Rüge ${ }^{26}$, bei der es sich um eine informelle Ermahnung handelt, steht bei ihnen nicht mehr der Appell, sich an die parlamentarischen Gepflogenheiten zu halten, im Vordergrund, sondern der Charakter eines formalen „Tadels“ beziehungsweise einer Missbilligung wegen einer nicht

22 Vgl. hierzu auch den dreitägigen Sitzungsausschluss der NPD-Fraktion aus dem Sächsischen Landtag vom 13. Juni 2012, LT-Plenarprotokoll $5 / 57$ vom 13. Juni 2012, S. 5730 f. (Tragen von Kleidung der Marke „Thor Steinar") sowie die Ablehnung eines Antrags auf Erlass einer einstweiligen Anordnung durch den Sächsischen Verfassungsgerichtshof vom 22. Juni 2012 - Az. Vf. 58-I-12, juris Rz. $33 \mathrm{ff}$.

23 Im Übrigen trägt der zur Begründung angeführte Verweis auf Hans Hugo Klein, in: Theodor Maunz / Günter Dürig, Grundgesetz, Stand 12/2007, Art. 40 Rz. 101, nicht. Dieser führt zwar aus, dass die Ordnungsgewalt allein den Zweck habe, den geordneten Ablauf der Plenarsitzung zu gewährleisten und nicht darüber hinaus reiche. Diese Aussage steht jedoch im Zusammenhang mit der Abgrenzung der Ordnungsgewalt vom Hausrecht. Sie ist eher so zu verstehen, dass das parlamentarische Ordnungsrecht das rechtliche Instrumentarium zur Wahrung der parlamentarischen Ordnung in den Sitzungen des Parlaments darstellt, während das Instrument, mittels dessen die Ordnung im parlamentarischen Raum außerhalb der Plenarsitzung aufrechtzuerhalten ist, das Hausrecht ist (so wohl auch die Interpretation der Fundstelle von Lars Brocker, in: Rudolf Dolzer I Karin Graßhof / Wolfgang Kahl / Christian Waldhoff (Hrsg.), a.a.O. (Fn. 11), Art 40 Rz. 131).

24 Vgl. Michael Köhler, a.a.O. (Fn. 9), S. 180 m.w.N.; Lars Brocker, a.a.O. (Fn. 11), Art 40 Rz. 118, Rz. 131; vgl. auch LVerfG Mecklenburg-Vorpommern, in: NordÖR 2009, S. 205, S. 207; VerfGH Sachsen, in: NVwZ-RR 2011, S. 129, S. 131.

25 BVerfGE 10, S. 4, S. 12; BVerfGE 60, S. 374, S. 379; Hans Hugo Klein, a.a.O. (Fn. 23), Art. 40 Rz. 103; Martin Brandt / Dieter Gosewinkel, Der Ausschluß eines Abgeordneten von der Plenarsitzung, in: ZRP, 19. Jg. (1986), S. 33 - 38, S. 33, S. 36. Entgegen der Auffassung von Albert Ingold / Sophie-Charlotte Lenski, a.a.O. (Fn. 8), gilt dies - wie noch zu zeigen sein wird - auch für das Ordnungsgeld.

26 Zur Rüge vgl. BVerfGE 60, S. 374, S. 379; Joseph Bücker, a.a.O. (Fn. 6), \$ 35 Rz. 15 f. 
hinnehmbaren Regelverletzung. ${ }^{27}$ Den damit verbundenen negativen Folgen hinsichtlich seines Ansehens in der Öffentlichkeit und im Parlament kann der Abgeordnete allein dadurch begegnen, dass er von seiner Redefreiheit eingeschränkten Gebrauch macht, indem er seine Wortwahl ändert beziehungsweise sein Verhalten im Plenum parlamentarischen Gepflogenheiten anpasst. ${ }^{28}$ Die Ordnungsmaßnahmen tangieren somit das durch Art. 38 Abs. 1 Satz 2 GG geschützte Rederecht, der Sitzungsausschluss darüber hinausgehend auch das Anwesenheits- und Stimmrecht.

Die Statusrechte unterliegen jedoch anerkanntermaßen den vom Parlament kraft seiner Geschäftsordnungsautonomie (Art. 40 Abs. 1 Satz 2 GG) gesetzten Schranken. Sofern also die in der Geschäftsordnung genannten Voraussetzungen der jeweiligen Ordnungsmaßnahmen vorliegen und dem Grundsatz der Verhältnismäßigkeit hinreichend Rechnung getragen wird, stoßen sie in verfassungsrechtlicher Hinsicht auf keine Bedenken. ${ }^{29}$ Im Übrigen fungiert die parlamentarische Ordnungsgewalt als notwendiges innerparlamentarisches Korrektiv gegenüber dem durch die Indemnität (Art. 46 Abs. 1 GG) gewährleisteten besonderen Schutz der parlamentarischen Redefreiheit. ${ }^{30}$ Dass ein Abgeordneter wegen seiner Äußerungen im Bundestag außerparlamentarisch hierfür nicht zur Verantwortung gezogen werden kann, erscheint unter anderem nur deshalb gerechtfertigt, weil das Parlament selbst mittels seiner Ordnungsgewalt Sorge dafür trägt, dass unakzeptable Äußerungen und Verhaltensweisen, die aber noch unter die Indemnität fallen, nicht von vornherein völlig sanktionslos gestellt werden.

\section{Katalog der formellen Ordnungsmaßnahmen und Entscheidungsspielraum des Präsidenten}

\subsection{Katalog der Ordnungsmaßnahmen}

Der um das Ordnungsgeld erweiterte und neu gefasste Katalog der formalen Ordnungsmaßnahmen sieht wie folgt aus:

- Sach- und Ordnungsruf (\$36 Abs. 1 GO-BT),

- Wortentziehung ( $\$ 36$ Abs. 2 GO-BT),

- Ordnungsgeld (\$37 GO-BT),

- Sitzungsausschluss ( $\$ 38$ GO-BT).

Die Möglichkeit, von informellen Maßnahmen wie der Rüge, Ermahnung oder Zurückweisung einer Äußerung als „,unparlamentarisch“ Gebrauch zu machen, bleibt von der Geschäftsordnungsänderung unberührt. Zudem kann nach wie vor bei störender Unruhe, die den Fortgang der Verhandlungen in Frage stellt, die Sitzung unterbrochen werden ( $\$ 40$ GO-BT).

Die Ordnungsmaßnahmen sehen hinsichtlich des Schweregrads der Ordnungsverletzung unterschiedliche Voraussetzungen vor. So verlangt $\$ 36$ Abs. 1 Satz 2 GO-BT für die Ertei-

27 Vgl. Ulrich Franke, Ordnungsmaßnahmen der Parlamente, Hamburg 1990, S. 75 m.w.N.

28 Vgl. Jürgen Milinski, Unbegrenztes „Rügerecht“ des Bundestagspräsidenten?, in: NJW 1983, H. 49, S. $2808-2809$.

29 Vgl. Joseph Bücker, a.a.O. (Fn. 6), \$34 Rz. 10; Michael Köhler, a.a.O. (Fn. 9), S. 178.

30 Vgl. Michael Köhler, a.a.O. (Fn. 9), S. 179; Joseph Bücker, a.a.O. (Fn. 6), \$34 Rz. 11; Hans-Peter Schneider, in: Alternativ Kommentar, Neuwied 1984, Art. 46 Rz. 8; Martin Brandt / Dieter Gosewinkel, a.a.O. (Fn. 25), S. 33, S. 36; vgl. auch LVerfG Mecklenburg-Vorpommern, in: NordÖR 2009, S. 205, S. 207. 
lung eines Ordnungsrufes lediglich, dass ein Mitglied des Bundestages „die Ordnung oder die Würde des Bundestages verletzt“. Demgegenüber verlangt die Festsetzung eines Ordnungsgeldes bereits „eine nicht nur geringfügige Verletzung der Ordnung oder der Würde des Bundestages“. Ein Ausschluss ist schließlich nur bei einer „gröblichen Verletzung “ möglich.

Die einzelnen Ordnungsmaßnahmen stehen zwar in einem Stufenverhältnis, gleichwohl aber selbständig nebeneinander. Es ist deshalb ohne weiteres möglich, direkt ein Ordnungsgeld festzusetzen, ohne dass zuvor ein Ordnungsruf erteilt worden ist beziehungsweise - wie bisher auch - einen Abgeordneten auszuschließen, ohne dass ein Ordnungsruf oder die Festsetzung eines Ordnungsgeldes dem vorangegangen wären. ${ }^{31}$ Sofern also nach Überzeugung des amtierenden Präsidenten der entsprechende Schweregrad für eine bestimmte Ordnungsmaßnahme vorliegt, kann er von ihr unmittelbar Gebrauch machen; es bedarf keines stufenweisen Vorgehens. Eine Ausnahme stellt insoweit allein der Wortentzug nach $\$ 35$ Abs. 3 und $\$ 36$ Abs. 2 GO-BT dar.

Wegen des unterschiedlichen Schweregrads der Ordnungsverletzung scheidet allerdings eine gleichzeitige Festsetzung eines Ordnungsgeldes und eines Sitzungsausschlusses aus. ${ }^{32}$ Auch für das Verhältnis von Ordnungsruf und Ordnungsgeld beziehungsweise Ordnungsruf und Sitzungsausschluss gilt im Grundsatz nichts anderes. Geht der Präsident lediglich von einer geringfügigen Ordnungsverletzung aus und erteilt dementsprechend dem Abgeordneten einen Ordnungsruf, so kommt daneben wegen desselben Sachverhalts die Festsetzung eines Ordnungsgeldes beziehungsweise eines Sitzungsausschlusses grundsätzlich nicht mehr in Betracht.

Etwas anderes gilt nur dann, wenn sich der amtierende Präsident bei Erteilung eines Ordnungsrufes ausdrücklich vorbehält, von der Möglichkeit einer nachträglichen Sanktion (Ordnungsgeld oder Ausschluss) Gebrauch zu machen. Hierin liegt keine doppelte Ahndung ein und desselben Vorgangs. Vielmehr schließt ein nachträglicher Sitzungsausschluss beziehungsweise die nachträgliche Festsetzung eines Ordnungsgeldes das Verfahren zur Reaktion auf eine Störung ab, die aufgrund der konkreten Umstände den amtierenden Präsidenten daran hindert, ad-hoc den Vorgang umfassend und abschließend geschäftsordnungsrechtlich zu bewerten. ${ }^{33}$

\subsection{Entscheidungsspielraum des Präsidenten}

Bei der Entscheidung, ob ein Verstoß gegen die Ordnung oder Würde des Bundestages vorliegt und wie schwer diese wiegt, steht dem Präsidenten ein Beurteilungsspielraum zu, da es sich bei den Tatbestandsmerkmalen der $\$ \$ 36$ ff. GO-BT um unbestimmte Rechtsbegriffe handelt. ${ }^{34}$ Davon zu trennen ist die Frage, ob der Präsident bei Vorliegen der entspre-

31 So explizit der Wortlaut der $\$ \$ 37$ und 38 GO-BT; Heinrich G. Ritzel / Joseph Bücker / Hermann J. Schreiner, a.a.O. (Fn. 5), $\$ 37$ Anmerkung 3 a); vgl. auch Lars Brocker, a.a.O. (Fn. 11), Art. 40 Rz. 134; anderer Auffassung LVerfG Mecklenburg-Vorpommern, in: NVwZ 2010, S. 958, S. 961.

32 BT-Drucksache 17/6309 vom 29. Juni 2011, S. 7.

33 Vgl. Heinrich G. Ritzel/ Joseph Bücker / Hermann J. Schreiner, a.a.O. (Fn. 5), \$ 37, Anmerkung 3 d) und $\$ 38$, Anmerkung $6 \mathrm{~d}$ ).

34 Vgl. Heinrich G. Ritzel / Joseph Bücker / Hermann J. Schreiner, a.a.O. (Fn. 5), Vorbemerkung zu $\$ \$ 36$ - 41, Anmerkung 4 b); Michael Köhler, a.a.O. (Fn. 9), S. 194; Ulrich Franke, a.a.O. (Fn. 27), S. 69; vgl. auch VerfGH Sachsen, in: NVwZ-RR 2011, S. 129, S. 132; in: NVwZ-RR 2012, S. 89, S. 90; LVerfG Mecklenburg-Vorpommern, in: NordÖR 2009, S. 205, S. 207; anderer Auffassung Albert Ingold / Sophie-Charlotte Lenski, a.a.O. (Fn. 8), S. 120, S. 122. 
chenden Voraussetzungen auch von dem jeweiligen Ordnungsmittel Gebrauch machen möchte. Dies steht in seinem pflichtgemäßen Ermessen, bei dessen Ausübung er sich einerseits von den Interessen an einem geordneten Sitzungsablauf sowie der Wahrung des Ansehens und der Würde des Parlaments in der Öffentlichkeit und andererseits von der Bedeutung der konkret betroffenen Statusrechte leiten lässt. ${ }^{35}$ Insbesondere gilt für die konkrete Anwendung der Ordnungsmaßnahmen der Opportunitätsgrundsatz. ${ }^{36}$ Der Präsident ist also nicht dazu gezwungen und kann auch nicht aus der Mitte des Hauses durch Antrag förmlich dazu angehalten werden, gegen eine Störung einzuschreiten. ${ }^{37}$ Er kann auch hierauf verzichten, zum Beispiel, weil Ablauf und Atmosphäre der jeweiligen Plenarsitzung dies tunlich erscheinen lassen und er eine weitere Zuspitzung der Situation vermeiden möchte. ${ }^{38}$

Mit der Anerkennung dieses weiten präsidialen Entscheidungsspielraums wird zu Recht - auch von der Rechtsprechung ${ }^{39}$ - dem Umstand Rechnung getragen, dass der Präsident in der Regel ad-hoc aus der Augenblickslage heraus zu reagieren hat. Die Sitzungsleitung im Plenum erfordert situationsbedingt eine schnelle Entscheidung („Sofortmaßnahme“), die im Interesse eines zügigen Fortgangs der parlamentarischen Beratungen möglichst im unmittelbaren zeitlichen Zusammenhang mit dem Ordnungsverstoß getroffen werden sollte. ${ }^{40}$ Insbesondere seitens der übrigen Abgeordneten, aber auch der Öffentlichkeit, wird regelmäßig eine unverzügliche Reaktion des amtierenden Präsidenten erwartet. Freilich erkennen die Geschäftsordnung und die parlamentarische Praxis an, dass dies nicht immer möglich ist, zum Beispiel weil die störenden Abgeordneten nicht sofort eindeutig identifizierbar sind oder der Sachverhalt noch einer genaueren Aufklärung bedarf. ${ }^{41}$ So besteht neben dem gewohnheitsrechtlich anerkannten nachträglichen Ordnungsruf ${ }^{42}$ für den Präsidenten auch die Möglichkeit, nachträglich ein Ordnungsgeld oder einen Sitzungsausschluss zu verhängen (\$\$ 37 S. 3, 38 Abs. 2 GO-BT).

Die besonderen Umstände ${ }^{43}$, unter denen der Präsident im Plenarsaal direkt zu entscheiden hat, und der hohe verfassungsrechtliche Status, der der Parlamentsautonomie zukommt, können dabei im Fall einer nachträglichen gerichtlichen Überprüfung von parlamentarischen Ordnungsmaßnahmen nicht unberücksichtigt bleiben. ${ }^{44}$

35 Vgl. Heinrich G. Ritzel / Joseph Bücker / Hermann J. Schreiner, a.a.O. (Fn. 5), Vorbemerkung zu $\$ \$ 36$ - 41, Anmerkung 4 b); Michael Köbler, a.a.O. (Fn. 9), S. 195.

36 Vgl. Lars Brocker, a.a.O. (Fn. 11), Art. 40 Rz. 134; Heinrich von Brentano, Die Rechtsstellung des Parlamentspräsidenten, Gießen 1930, S. 44.

37 Vgl. Michael Köhler, a.a.O. (Fn. 9), S. 195 m.w.N.

38 Vgl. Heinrich G. Ritzel / Joseph Bücker / Hermann J. Schreiner, a.a.O. (Fn. 5), Vorbemerkung zu $\$ \$ 36-41$, Anmerkung 4 b).

39 Vgl. etwa VerfGH Sachsen, in: NVwZ-RR 2012, S. 89, S. 90; Urteil vom 3. Dezember 2010 - Vf. 16-I-10, juris Rz. 21.

40 So ausdrücklich VerfGH Sachsen, Urteil vom 3. Dezember 2010 - Vf. 16-I-10, juris Rz. 21.

41 Vgl. Heinrich G. Ritzel / Joseph Bücker / Hermann J. Schreiner, a.a.O. (Fn. 5), \$ 38, Anmerkung 6 a); BT-Drucksache 16/13492 vom 18. Juni 2009, S. 4 ff.

42 Vgl. Heinrich G. Ritzel / Joseph Bücker / Hermann J. Schreiner, a.a.O. (Fn. 5), \$36, Anmerkung 3 i).

43 Hierzu gehört auch, dass trotz Protokoll und Fernsehaufzeichnungen die konkrete Situation und Stimmungslage im Plenarsaal im Nachhinein nur begrenzt nachempfunden werden kann. Die gleiche Bemerkung oder der gleiche Zwischenruf kann je nach Situation völlig unterschiedlich wirken.

44 Zur verfassungsgerichtlichen Kontrolldichte vgl. Lars Brocker, a.a.O. (Fn. 11), Art. 40 Rz. 368; Michael Köhler, a.a.O. (Fn. 9), S. 222; Ulrich Franke, a.a.O. (Fn. 27), S. 146, die zum Teil eine Beschränkung auf Willkürakte des Präsidenten annehmen. Zurückhaltend in ähnlichem Zusam- 


\section{Ordnungsgeld nach $\ 37$ GO-BT}

Nach $\$ 37$ GO-BT kann der Präsident wegen einer nicht nur geringfügigen Verletzung der Ordnung oder der Würde des Bundestages gegen ein Mitglied des Bundestages, auch ohne dass ein Ordnungsruf ergangen ist, ein Ordnungsgeld in Höhe von 1.000 Euro festsetzen. Im Wiederholungsfall erhöht sich das Ordnungsgeld auf 2.000 Euro.

Das Ordnungsgeld als zusätzliches, mit finanziellen Sanktionen verbundenes Instrument der Ordnungsgewalt orientiert sich an Vorbildern und historischen Vorläufern, die sich unter anderem im Geschäftsordnungsrecht des Europäischen Parlaments, der französischen Nationalversammlung und des saarländischen Landtages finden. ${ }^{45}$

\subsection{Verfassungsrechtliche Einwände gegen das Ordnungsgeld}

Gegen die Einführung des Ordnungsgeldes werden grundsätzliche verfassungsrechtliche Einwände erhoben. ${ }^{46}$ Ingold und Lenski ${ }^{47}$ sehen im Ordnungsgeld wegen der mit der finanziellen Sanktion verbundenen Auswirkung auf die persönliche Rechtsstellung des Abgeordneten eine zur Aufrechterhaltung der Arbeits- und Funktionsfähigkeit des Parlaments nicht erforderliche und deshalb unverhältnismäßige verfassungswidrige Einschränkung des freien Mandats. Darüber hinaus soll die starre Pauschalierung der Geldbeträge einen Verstoß gegen das rechtsstaatliche Schuldprinzip darstellen. ${ }^{48}$

Zur Begründung, dass das Ordnungsgeld einen unverhältnismäßigen Eingriff in die durch Artikel 38 Abs. 1 Satz 2 GG geschützten Rechtspositionen des Abgeordneten darstellt, unterscheiden Ingold und Lenski zunächst zwischen Eingriffen in die so genannten Statusrechte (vor allem Rede-, Anwesenheits- und Stimmrecht) und solchen in das freie Mandat, das heißt in die persönliche Unabhängigkeit des Abgeordneten. Zwischen beiden Eingriffsarten wird ein Rangverhältnis behauptet, wonach Eingriffe in die parlamentarischen Befugnisse und Rechte weniger schwer wiegen sollen als solche in das freie Mandat. Im Unterschied zu den herkömmlichen Ordnungsmaßnahmen (Ordnungsruf, Wortentzug und Ausschluss), bei denen „nur“ die Statusrechte des Abgeordneten berührt seien, treffe das Ordnungsgeld den Abgeordneten nicht als Mandatsträger, sondern allein als Privatperson. ${ }^{49}$ Da die Sanktionswirkung den Innenbereich des Parlaments verlasse, gehe es mithin nicht mehr nur um eine im Binnenbereich des Bundestages vorgenommene „bloße“ Ausgestaltung

menhang auch BVerfGE 99, S. 19, S. 34; anderer Auffassung Albert Ingold / Sophie-Charlotte Lenski, a.a.O. (Fn. 8), S. 120, S. 122; vgl. auch LVerfG Mecklenburg-Vorpommern, in: NordÖR 2009, S. 205, S. 208, mit ablehnender Anmerkung von Wilfried Erbguth / Mathias Schubert.

45 Art. 153 Geschäftsordnung Europäisches Parlament; Art. 76 Geschäftsordnung Französische Nationalversammlung; $\$ 73$ Gesetz über den Landtag des Saarlandes. Im Zusammenhang mit dem Sitzungsausschluss kannten auch einzelne Geschäftsordnungen früherer Landesparlamente (Bremen, Hessen, Mecklenburg-Schwerin und Thüringen) finanzielle Sanktionen wie zum Beispiel den Verlust der Diäten oder der Freifahrtkarte für die Dauer des Ausschlusses, vgl. hierzu Heinrich von Brentano, a.a.O. (Fn. 36), S. 51 ff.

46 Speziell zur Kritik an dem Begriff der „Würde“ sogleich unter 3.2.

47 Vgl. Albert Ingold/ Sophie-Charlotte Lenski, a.a.O. (Fn. 8), S. 120, S. 124.

48 Dazu unter 3.4.

49 Vgl. Albert Ingold / Sophie-Charlotte Lenski, a.a.O. (Fn. 8), S. 120, S. 125. 
der Statusrechte der Abgeordneten, sondern um eine schwerer wiegende Einschränkung der persönlichen Unabhängigkeit und damit des freien Mandats. ${ }^{50}$

Schon die Differenzierung zwischen ordnungsrechtlichen Eingriffen in Statusrechte einerseits und in das freie Mandat andererseits vermag nicht zu überzeugen. So ist es nicht plausibel, bei einem Ordnungsruf von einem Eingriff in die verfassungsrechtlich gewährleisteten Statusrechte des Abgeordneten, insbesondere in das Rederecht, auszugehen, dies aber bei dem schärferen Sanktionsinstrument des Ordnungsgeldes, mit dem der Präsident eine schwerer wiegende Ordnungsverletzung feststellt, zu verneinen. Zwar ist es richtig, dass das Ordnungsgeld den Abgeordneten wegen der finanziellen Einbuße (zum Beispiel in Gestalt einer denkbaren Verrechnung mit Ansprüchen auf Entschädigung nach $\$ 11 \mathrm{AbgG}$ ) in seinem privaten Rechtskreis berührt; das Ordnungsgeld trifft ihn jedoch nicht in erster Linie, sondern auch als Privatperson. Die „Ahndung“ des Fehlverhaltens im Plenum, dessen offizielle und formelle Missbilligung durch den Präsidenten, steht nach wie vor im Vordergrund. Ordnungsgeld und Ordnungsruf unterscheiden sich insoweit allein durch die zusätzliche Ausstrahlungswirkung des Ordnungsgeldes auf die persönliche Rechtsstellung des Abgeordneten. Die Prämisse, der Abgeordnete werde durch das Ordnungsgeld ausschließlich als Privatperson und nicht in seinen parlamentarischen Befugnissen als Mandatsträger tangiert, trifft deshalb nicht zu.

Unbegründet bleibt auch, warum die finanzielle Sanktion unmittelbar das freie Mandat berühren soll. Das freie Mandat dient in erster Linie dem Schutz des Abgeordneten gegen den Verlust des Mandats und gegen zwangsweise Einflussnahmen auf die Art seiner Ausübung. ${ }^{51}$ Keine gesetzliche Regelung darf dem Abgeordneten vorschreiben, was er im Bundestag zu sagen hat, wie er im Ausschuss arbeitet oder im Plenum abstimmt, oder solches Verhalten in irgendeiner Weise rechtsförmig sanktionieren. ${ }^{52}$ Durch die Zahlung eines Ordnungsgeldes wird der Abgeordnete jedoch nicht daran gehindert, unabhängig und frei nach seinem Gewissen zu entscheiden und sich dementsprechend zu verhalten. Das Ordnungsgeld richtet sich - ebenso wie alle anderen Ordnungsmaßnahmen - gegen bestimmte, nicht tolerable äußere Formen des Verhaltens im Plenum, es zielt aber nicht auf die inhaltliche Wahrnehmung des Mandats. Darin liegt gerade ein entscheidender Vorteil des Ordnungsgeldes: Anders als beim Sitzungsausschluss ist eben nicht zu befürchten, dass die Wahrnehmung des Mandats - etwa durch „Versäumen“ einer Abstimmung - tangiert wird.

Ingold und Lenski scheinen dies durchaus zu konstatieren, sehen aber offenbar unter Hinweis auf die Entscheidung des Bundesverfassungsgerichts zu den Nebeneinkünften der Abgeordneten ${ }^{53}$ die wirtschaftliche Unabhängigkeit des Abgeordneten als von der Schutzwirkung des Art. 38 Abs. 1 Satz 2 GG mit umfasst an. ${ }^{54}$ In der Tat dient die Entschädigung der materiellen Absicherung des freien Mandats. Art. 48 Abs. 3, Satz 1 GG sichert die Freiheit des Abgeordneten ergänzend $\mathrm{ab}$ und gewährleistet so die tatsächliche Grundlage für dessen Unabhängigkeit. ${ }^{55}$ Das bedeutet jedoch nicht, dass Abgeordnetenpflichten, zu denen auch

50 Vgl. ebenda, S. 120, S. 125.

51 Vgl. Martin Morlok, in: Horst Dreier, Grundgesetz, 2. Aufl. 2006, Art. 38 Rz. 142 m.w.N.

52 Vgl. Christoph Möllers, Das freie Mandat in der demokratischen Repräsentation - BVerfGE 118, 277 - Urteil vom 4. Juli 2007 (2 BvE 1-4/06), in: Jura 2008, S. 937 - 942, S. 937, S. 940.

53 BVerfGE 118, S. 277 ff.; zur Entscheidung vgl. Christoph Möllers, a.a.O. (Fn. 52), S. 937 ff.

54 Vgl. Albert Ingold / Sophie-Charlotte Lenski, a.a.O. (Fn. 8), S. 120, S. 122.

55 Vgl. Martin Morlok, in: Horst Dreier, a.a.O. (Fn. 51), Art. 38 Rz. 148. 
die Einhaltung der vom Parlament selbst gesetzten Regeln zählen, nicht auch mit „rechtsförmigen Sanktionen “ bewehrt, sondern allein über die politische Verantwortung gegenüber dem Wähler reguliert sein dürfen. ${ }^{56}$ Der Abgeordnetenstatus ist - wie das Bundesverfassungsgericht zutreffend betont - „nicht prinzipiell sanktionsfrei ausgestaltet“57. Das Parlament ist vielmehr dazu berechtigt, im Interesse seiner Funktionsfähigkeit die selbst gesetzten Regelungen im Bedarfsfall auch mit Sanktionen durchzusetzen. Die repräsentative Funktion des freien Mandats beruht auf der Einhaltung einer Vielzahl von Organisations- und Verfahrensregeln, die erst in ihrem Zusammenspiel das Repräsentationsprinzip zur Geltung bringen. „Das freie Mandat schließt deshalb die Möglichkeit, die zu seiner institutionellen Entfaltung erforderlichen Sanktionen zu verhängen, nicht aus, sondern ein." 58

Damit ist die Frage aufgeworfen, unter welchen Voraussetzungen finanzielle Sanktionen als Eingriff in Artikel 38 Abs. 1 Satz 2 GG gerechtfertigt sein können. Ingold und Lenski betonen in diesem Zusammenhang einen unterschiedlichen verfassungsrechtlichen Prüfungsmaßstab bei Eingriffen in Statusrechte und solchen in das freie Mandat. Sie nehmen insoweit ein Rangverhältnis an, das dazu zwingen soll, Fragen der parlamentarischen Selbstorganisation zunächst durch Ausgestaltungen der Statusrechte zu lösen, bevor auf Einschränkungen des freien Mandats zurückgegriffen werden kann. Dies sei nicht geschehen, obwohl der mit dem Ordnungsgeld verfolgte Zweck - die Wiederherstellung der Funktionsfähigkeit des Parlaments - auch durch die rein innerparlamentarisch wirkenden Maßnahmen des Ordnungsrufs oder Sitzungsausschlusses hätten herbeigeführt werden können.

Unabhängig davon, dass das Rangverhältnis lediglich behauptet, aber nicht begründet wird $^{59}$, legen Ingold und Lenski nicht ansatzweise dar, wie die mit dem Ordnungsgeld beabsichtigte Zielsetzung, dem sitzungsleitenden Präsidenten eine angemessenere Handhabung der Ordnungsgewalt im Einzelfall zu ermöglichen, konkret erreicht werden kann, dies um so weniger, als aufgrund eines restriktiven Verständnisses des Ordnungsbegriffs eine Vielzahl von Störungen gar nicht der Ordnungsgewalt unterfallen sollen. Berücksichtigt man zudem, dass nach Auffassung von Ingold und Lenski der Sitzungsausschluss weder den Entzug des Stimmrechts nach sich ziehen noch mit Wirkung über den Sitzungstag hinaus verhängt werden darf ${ }^{60}$, führt dies in einem Maße zu Beschränkungen der Handlungsmöglichkeiten des Präsidenten, die die Aufrechterhaltung einer ordnungsgemäßen Sitzung ernsthaft in Frage stellen. Bei Licht betrachtet, bleibt dem Präsidenten somit nicht mehr als ein einfacher Ordnungsruf und ein zu einer Art Saalverweis „mutierter“ Ausschluss, der zu Abstimmungen hin unterbrochen werden müsste. Da im Bundestag im Rahmen des jeweiligen Tagesordnungspunktes abgestimmt wird, es also grundsätzlich keine „gebündelten“ Abstimmungen gibt, würde eine derartige Anwendung des $\$ 38$ GO-BT lächerlich wirken. ${ }^{61}$ Nach alledem greifen die vorgebrachten verfassungsrechtlichen Einwände gegen das Ordnungsgeld nicht durch.

56 BVerfGE 118, S. 277, S. 374.

57 Ebenda, S. 375.

58 Ebenda; Beispiele für anerkannte Sanktionen nennt Gerald Kretschmer, in: Bruno Schmidt-Bleibtreu / Hans Hofmann / Axel Hopfauf, Grundgesetz, 12. Aufl. 2011, Art. 48 Rz. 27.

59 Vgl. Albert Ingold / Sophie-Charlotte Lenski, a.a.O. (Fn. 8), S. 120, S. 125, „spricht jedoch vieles dafür, zwischen Ausgestaltungen der Statusrechte und Beeinträchtigungen des freien Mandats ein Rangverhältnis anzunehmen".

60 Vgl. Albert Ingold/ Sophie-Charlotte Lenski, a.a.O. (Fn. 8), S. 120, S. 123 f.

61 Vgl. Heinrich G. Ritzel / Joseph Bücker / Hermann J. Schreiner, a.a.O. (Fn. 5), \$38, Anmerkung $2 \mathrm{c})$. 


\subsection{Verletzung der Ordnung oder der Würde des Bundestages}

Im Tatbestand des Ordnungsgeldes wird ebenso wie bei der Neufassung des Ordnungsrufes und Ausschlusses ausdrücklich die „Würde des Bundestages“ erwähnt. Damit geht indes keine Ausweitung des Schutzbereichs einher, da die Verletzung der „Würde“ des Bundestages schon bisher im Rahmen der Geschäftsordnung stets als eine Ordnungsverletzung im Sinne der $\$ \$ 36$ ff. GO-BT angesehen wurde und überdies eine sinnvolle und trennscharfe Unterscheidung nicht möglich ist. ${ }^{62}$ Durch die Ergänzung soll lediglich deutlicher zum Ausdruck kommen, dass auch „nicht verbale Ordnungsstörungen, wie zum Beispiel das Hochhalten von Transparenten, das Tragen von Ansteckplaketten je nach Gegebenheiten und Inhalten oder sonstiges provokatives Verhalten, eine Verletzung der Würde des Bundestages darstellen können" 63 . Reine Fragen der Kleiderordnung sollen allerdings hiervon ausgenommen sein, sofern nicht im Einzelfall allgemeine Regeln des Anstands verletzt werden. ${ }^{64}$

Die Aufnahme des Begriffs der „Würde“ war im Rahmen der parlamentarischen Beratungen nicht unumstritten. Insbesondere die Fraktion Bündnis 90/Die Grünen kritisierte, dass die Einführung dieser „neuen Tatbestandsalternative“ nicht mehr, sondern weniger Klarheit und Rechtssicherheit schaffe und zudem die Gefahr in sich berge, Geschmacksfragen zu sanktionieren. ${ }^{65}$ Auch sei es grundsätzlich problematisch, den Begriff der Würde auf eine Institution anzuwenden, statt wie üblich sie im Zusammenhang mit Individuen zu verwenden. ${ }^{66}$ Gleichlautende Argumente finden sich in Teilen des Schrifttums. ${ }^{67}$ Auch dort wird insbesondere moniert, dass Kategorien wie die Würde und das Ansehen des Parlaments viel zu unbestimmt seien, um im Interesse einer rationalen und nachvollziehbaren Rechtsanwendung Einschränkungen der Statusrechte der Abgeordneten mit zum Teil repressiver Wirkung rechtfertigen zu können.

Dem ist entgegenzuhalten, dass der Bundestag mit der expliziten Erwähnung der Würde keineswegs parlamentsrechtliches Neuland betritt beziehungsweise einen „Sonderweg“ beschreitet. Bereits die Geschäftsordnung des Preußischen Abgeordnetenhauses sah in $\$ 64$ Abs. 2 vor, dass „im Falle besonders grober, die Würde des Hauses schädigender Verletzung der Ordnung“ jemand für den Rest der Sitzung ausgeschlossen werden konnte. ${ }^{68}$ Im Bundestag selbst ist in der parlamentarischen Praxis nie in Frage gestellt worden, dass auch Verletzungen der Würde ordnungsrechtliche Sanktionen nach sich ziehen können. ${ }^{69}$ Auf der

62 Beispiele bei Heinrich G. Ritzel / Joseph Bücker / Hermann J. Schreiner, a.a.O. (Fn. 5), Vorbemerkung zu $\$ \$ 36-41$, Anmerkung 1d).

63 BT-Drucksache 17/5471 vom 12. April 2011, S. 4; BT-Drucksache 17/6309 vom 29. Juni 2011, S. 5.

64 BT-Drucksache 17/5471 vom 12. April 2011, S. 4; zum Hintergrund vgl. Heinrich G. Ritzel / Joseph Bücker / Hermann J. Schreiner, a.a.O. (Fn. 5), Vorbemerkung zu $\$ \$ 36$ - 41, Anmerkung $1 \mathrm{~d})$.

65 BT-Drucksache 17/6352 vom 29. Juni 2011.

66 BT-Drucksache 17/6309 vom 29. Juni 2011, S. 6.

67 Vgl. Martin Brandt / Dieter Gosewinkel, a.a.O. (Fn. 25), S. 33, S. 36; Ulrich Franke, a.a.O. (Fn. 27), S. 68 f., S. 109; Albert Ingold / Sophie-Charlotte Lenski, a.a.O. (Fn. 8), S. 120, S. 122 f.

68 Vgl. Heinrich G.Ritzel / Joseph Bücker / Hermann J. Schreiner, a.a.O. (Fn. 5), Vorbemerkung zu $\$ \$ 36$ - 41, Anmerkung 1d).

69 Zum Beispiel anlässlich der bei Heinrich G. Ritzel / Joseph Bücker / Hermann J. Schreiner, a.a.O. (Fn. 5), Vorbemerkung zu $\$ \$ 36$ - 41, Anmerkung 1d) genannten Fälle. 
Ebene der Bundesländer wird die Würde als Schutzgut der Ordnungsgewalt in den Geschäftsordnungen der Landtage von Hessen, Mecklenburg-Vorpommern, Nordrhein-Westfalen, Rheinland-Pfalz und Thüringen ausdrücklich erwähnt. ${ }^{70}$ Gleiches gilt im internationalen Rahmen, beispielsweise für das Europäische Parlament ${ }^{71}$ oder den Österreichischen Nationalrat. ${ }^{72}$

Es überzeugt auch nicht, Institutionen generell einen Schutz ihrer Würde beziehungsweise ihres Ansehens zu versagen und auf Personen beschränken zu wollen. So nimmt $\$ 7$ Abs. 1 Satz 2 GO-BT den Bundestagspräsidenten zur Wahrung der Würde und der Rechte des Bundestages ausdrücklich in die Pflicht. ${ }^{73}$ Auch außerhalb des Parlamentsrechts besteht anerkanntermaßen ein institutioneller Schutz, zum Beispiel zur Wahrung des Ansehens der Gerichte. So sieht $\$ 178$ GVG vor, dass gegen Teilnehmer einer Gerichtsverhandlung, die sich in der Sitzung einer Ungebühr schuldig machen, mit Ordnungsmaßnahmen vorgegangen werden kann. Darunter fallen auch Verhaltensweisen, die zwar nicht den äußeren Sitzungsverlauf beeinträchtigen, aber den notwendigen Respekt vor dem Gericht vermissen lassen. Dies wird im Einzelfall auch bei einer „provozierenden“ Kleidung angenommen. ${ }^{74}$ Insoweit reklamiert das Parlament nichts Anderes oder Weitergehendes für sich. ${ }^{75}$

Da Regelverstöße im Parlament wegen des mit ihnen verbundenen Akzeptanz- und Ansehensverlustes auf die Institution als solche zurückfallen können, obliegt es zu Recht dem Parlament selbst, Inhalt und Grenzen der in ihm akzeptierten Verhaltensweisen festzulegen und zu entscheiden, wie lange und in welchem Umfang diese gelten sollen. Änderungen der zugrunde liegenden Wertungen können dabei nur über einen veränderten Konsens im Parlament, nicht aber allein durch ein nonkonformes Verhalten einzelner Mitglieder herbeigeführt werden. Eine, wie von Ingold und Lenski ${ }^{76}$ behauptete, Zementierung einmal akzeptierter Verhaltensweisen, die sich gegen eine „Fortentwicklung der Selbstverständnisbildung“ immun zeigt, ist damit nicht verbunden, denn über Verhaltensweisen und Äußerungsformen sowie einem damit eventuell verbundenen Wandel kann in parlamentarischen Gremien wie dem Ältestenrat oder Präsidium debattiert und eine Verständigung zu erreichen versucht werden. Zudem besteht über die Möglichkeit eines Einspruchs nach $₫ 39$ GO-BT für jedes von einer Ordnungsmaßnahme betroffene Mitglied die Chance, die Mehrheit der Abgeordneten für eine Aufhebung der Sanktion zu gewinnen, um auf diesem in der Geschäftsordnung vorgesehenen Verfahrensweg zu einem veränderten Selbstverständnis des Parlaments

$70 \$ 75$ Abs. 2 GO-LT-Hessen; $\$ 97$ Abs. 2 GO-LT-MV; $\$ 34$ Abs. 2 GO-LT-NRW; $\$ 39$ Abs. 1 GO-LT-RP; $\$ 37$ Abs. 1 GO-LT-Thüringen.

71 Art. 9 Abs. 2 i.V.m. 152 ff. Geschäftsordnung EP, 7. Wahlperiode (Stand: April 2012).

$72 \$ 102$ Abs. 2 Bundesgesetz über die Geschäftsordnung des Nationalrats.

73 Vgl. Lars Brocker, a.a.O. (Fn. 11), Art. 40 Rz. 118; vgl. hierzu auch den Redebeitrag des Abgeordneten Thomas Strobl, BT-Plenarprotokoll 17/117 vom 30. Juni 2011, S. 13592 D.

74 Vgl. Walter Zimmermann, in: Thomas Rauscher / Peter Wax / Joachim Wenzel (Hrsg.), Münchener Kommentar zur Zivilprozessordnung, München 2008, \$178 GVG Rz. 4 ff. Als Beispiele hierfür werden das Erscheinen im Badeanzug und Entblößen genannt (Rz. 8).

75 Vgl. hierzu auch den dreitägigen Sitzungsausschluss der NPD-Fraktion aus dem Sächsischen Landtag vom 13. Juni 2012, LT-Plenarprotokoll $5 / 57$ vom 13. Juni 2012, S. 5730 f (Tragen von Kleidung der Marke „Thor Steinar") sowie die Ablehnung eines Antrags auf Erlass einer einstweiligen Anordnung durch den Sächsischen Verfassungsgerichtshof vom 22. Juni 2012 - Az. Vf. 58-I-12, juris.

76 Vgl. Albert Ingold / Sophie-Charlotte Lenski, a.a.O. (Fn. 8), S. 120, S. 123. 
beizutragen. Im Einklang mit der Rechtsprechung und weiten Teilen der Literatur, die die Würde des Hauses als Schutzgut der Ordnungsgewalt ansehen, greifen deshalb die vorgebrachten verfassungsrechtlichen Bedenken gegen die im Geschäftsordnungstext erfolgte Klarstellung nicht durch. ${ }^{77}$

\subsection{Schwere der Ordnungsverletzung}

Das Ordnungsgeld kann wegen einer „nicht nur geringfügigen“ Verletzung der Ordnung oder der Würde des Bundestages festgesetzt werden. Es muss sich also um eine Ordnungsverletzung von einer gewissen Intensität handeln, für die einerseits ein bloßer Ordnungsruf nicht ausreichend erscheint, die andererseits aber noch nicht als gröbliche Verletzung gewertet wird. Ob im konkreten Fall eine „nicht nur geringfügige Verletzung der Ordnung oder der Würde des Bundestages" vorliegt, unterliegt, da es sich um unbestimmte Rechtsbegriffe handelt, dem Beurteilungsspielraum des amtierenden Präsidenten.

Der Geschäftsordnungsgeber hat eine nähere Konkretisierung, zum Beispiel durch die Nennung von Fallgruppen, als wenig sinnvoll erachtet, da dies ebenso wenig eine Diskussion im Einzelfall unterbinden würde wie die weitere Umschreibung durch unbestimmte Rechtsbegriffe. ${ }^{78}$ Als Maßstab könne aber der Umfang der Beeinträchtigung der Rechte der übrigen Mitglieder des Bundestages, insbesondere auf möglichst ungestörte Verfolgung der Plenardebatte oder des ungestörten Rederechts, herangezogen werden ${ }^{79}$.

In der Praxis dürfte sich eine Orientierung an den bisherigen Ausschlussfällen nach dem Grundsatz anbieten: „Im Zweifel Ordnungsgeld statt Ausschluss. “80 Dies gilt insbesondere für Fälle demonstrativer Störungen, die im Wesentlichen auch den Anlass für die Einführung des Ordnungsgeldes gebildet haben (Transparente, Spruchbänder, Tragen einheitlicher Kleidungsstücke mit Aufdruck etc.). ${ }^{81}$ Bei dieser Regel kann es sich jedoch nur um eine grobe Richtschnur handeln, da mit der Einführung des Ordnungsgeldes eine neue Abstufung und Gewichtung der Ordnungsmittel einher gegangen ist. Der Präsident ist deshalb gehalten, in jedem Einzelfall zu prüfen, ob in Konstellationen, bei denen bislang ein Sitzungsausschluss für zulässig erachtet wurde, das Ordnungsgeld nicht die angemessenere und sinnvollere Sanktion darstellt, da es nicht mit einem Entzug des Stimmrechts oder mit öffentlichkeitswirksamen Konfrontationen bis hin zu einer zwangsweisen Entfernung aus dem Plenarsaal verbunden ist. ${ }^{82}$ Nach Einführung des Ordnungsgeldes sollte ein Ausschluss mehr denn je nur „ultima ratio“ sein.

77 Vgl. Heinrich G. Ritzel / Joseph Bücker / Hermann J. Schreiner, a.a.O. (Fn. 5), Vorbemerkung zu $\$ \$ 36$ - 41, Anmerkung 1 a); Lars Brocker, a.a.O. (Fn. 11), Art. 40 Rz. 118; Gerhard Weng, a.a.O. (Fn. 17), S. 248, S. 255; vgl. auch LVerfG Mecklenburg-Vorpommern, in: NordÖR 2009, S. 205, S. 207; VerfGH Sachsen, in: NVwZ-RR 2011, S. 129, S. 131.

78 BT-Drucksache 17/6309 vom 29. Juni 2011, S. 6.

79 Ebenda.

80 Heinrich G. Ritzel / Joseph Bücker / Hermann J. Schreiner, a.a.O. (Fn. 5), \$37, Anmerkung 2 b) und $\$ 38$, Anmerkung 2b); BT-Drucksache 16/13492 vom 18. Juni 2009, S. 4.

81 Vgl. Heinrich G. Ritzel/ Joseph Bücker / Hermann J. Schreiner, a.a.O. (Fn. 5), §37, Anmerkung 2 b); BT-Drucksache 17/5471 vom 12. April 2011, S. 3.

82 Vgl. Heinrich G. Ritzel / Joseph Bücker / Hermann J. Schreiner, a.a.O. (Fn. 5), \$ 37, Anmerkung 2 b) und $\$ 38$, Anmerkung $2 \mathrm{~b}$ ). 


\subsection{Höhe des Ordnungsgeldes}

Ob der Präsident bei einer „nicht nur geringfügigen“ Verletzung der Ordnung von der Möglichkeit der Festsetzung eines Ordnungsgeldes Gebrauch macht, liegt in seinem Ermessen. Dies gilt nicht für die Höhe des Ordnungsgeldes, da dies auf 1.000 Euro festgeschrieben ist, um Streitigkeiten hierüber im Einzelfall zu vermeiden. ${ }^{83}$

Ingold und Lenski ${ }^{84}$ sehen hierin eine „starre Pauschalierung“, die mit dem verfassungsrechtlichen Schuldprinzip nicht vereinbar ist. Danach müsse das Maß der individuellen Schuld den Rahmen für die Strafzumessung im konkreten Fall unter Beachtung der Strafzwecke und des Verhältnismäßigkeitsgrundsatzes bilden. Dies gelte nicht nur im eigentlichen Strafrecht, sondern auch für strafähnliche Sanktionen und für Disziplinarmaßnahmen. Da Ordnungsmaßnahmen wie der Sitzungsausschluss und das Ordnungsgeld unstreitig strafähnlichen Charakter hätten und Verschulden voraussetzten, bestehe auch bei ihnen eine strikte Bindung an das rechtsstaatliche Schuldprinzip.

Die Kritik übersieht, dass bereits der gestufte Katalog möglicher Ordnungsmaßnahmen, ob formeller oder informeller Natur, ein verhältnismäßiges, den Gegebenheiten des Einzelfalles angemessenes Einschreiten ermöglicht. ${ }^{85}$ Das Ordnungsgeld darf nicht isoliert betrachtet werden, es muss vielmehr stets im Zusammenhang mit den anderen Ordnungsmitteln gesehen werden. ${ }^{86}$ Von besonderer Bedeutung ist hierbei, dass das parlamentarische Ordnungsrecht nicht über ein System besonderer Tatbestände verfügt, es kennt nur ein einziges „Delikt“: die Verletzung der „Ordnung oder der Würde des Bundestages“. Entscheidet der Präsident sich in Kenntnis der Festbeträge für ein Ordnungsgeld, so liegt dem bereits eine hinreichende Betrachtung und Bewertung der besonderen Umstände des Einzelfalles, insbesondere der Schwere des Verstoßes und der persönlichen Vorwerfbarkeit zugrunde. Dies lässt die Einräumung eines weiteren Ermessens bezogen auf die Höhe der Zahlung entbehrlich erscheinen, zumal der Geschäftsordnungsgeber mit den festen Geldbeträgen Ungleichbehandlungen vermeiden wollte. ${ }^{87}$ Zudem darf nicht verkannt werden, dass es sich bei der Ordnungsgewalt - trotz ihres teilweise pönalen Charakters - nicht um eine Abart der staatlichen Strafgewalt handelt. Die $\$ \$ 36$ ff. GO-BT stellen kein Sonderstrafrecht dar. ${ }^{88}$ Die Ordnungsgewalt ist weitgehend durch das Opportunitätsprinzip und nicht durch das Legalitätsprinzip gekennzeichnet. ${ }^{89}$

83 BT-Drucksache 17/6309 vom 29. Juni 2011, S. 6; Heinrich G. Ritzel / Joseph Bücker / Hermann J. Schreiner, a.a.O. (Fn. 5), $\$ 37$, Anmerkung 3 b).

84 Vgl. Albert Ingold / Sophie-Charlotte Lenski, a.a.O. (Fn. 8), S. 120, S. 124.

85 Vgl. Wilfried Erbguth / Mathias Schubert, in: NordÖR 2009, S. 209, S. 210.

86 Vgl. auch den Redebeitrag des Abgeordneten Bernhard Kaster, BT-Plenarprotokoll 17/117 vom 30. Juni 2011, S. 13524 A.

87 BT-Drucksache 17/6309 vom 29. Juni 2011, S. 6.

88 Vgl. Michael Köhler, a.a.O. (Fn. 9), S. 179.

89 Vgl. Heinrich von Brentano, a.a.O. (Fn. 36), S. 44; Lars Brocker, a.a.O. (Fn. 11), Art. 40 Rz. 134, S. 44; Michael Köhler, a.a.O. (Fn. 9), S. 179. 


\subsection{Besonderheit: Wiederholungsfall}

In der spezifischen Ausgestaltung einer Sanktion für Wiederholungsfälle findet das System abgestufter Sanktionsmöglichkeiten des Präsidenten einen weiteren besonderen Ausdruck, denn bei einer wiederholten Störung ist er trotz der darin sichtbar werdenden Missachtung geltender Regelungen und einer fortgesetzten Infragestellung seiner Autorität nicht zum Sitzungsausschluss gezwungen, um „angemessen“ zu reagieren.

Das Abgeordnetengesetz und die Geschäftsordnung enthalten zwar keine Definition des Wiederholungsfalls, nach dem Willen des Geschäftsordnungsgebers soll dieser jedoch in der Regel dann vorliegen, wenn das betroffene Mitglied des Bundestages innerhalb von drei Sitzungswochen erneut Anlass für die Festsetzung eines Ordnungsgeldes aus ähnlichen Gründen gegeben hat. ${ }^{90}$ Voraussetzung für das Vorliegen eines Wiederholungsfalls ist danach zunächst, dass derselbe Abgeordnete zuvor eine Plenarsitzung gestört hat. Die Störung durch ein anderes Mitglied derselben Fraktion genügt nicht, da eine Zurechnung fremden Verhaltens ausscheidet. Die vorangegangene Störung muss ebenfalls mit einem Ordnungsgeld und nicht mit einer anderen Ordnungsmaßnahme geahndet worden sein. Die Gründe, die den Anlass für die Festsetzung der Ordnungsgelder gegeben haben, müssen „ähnlich“ sein, die zugrunde liegenden Sachverhalte müssen also im Wesentlichen vergleichbar sein. Dies dürfte bei wiederholten Meinungskundgaben per Transparent oder bedruckter Kleidung nahe liegen, nicht aber bei Zuwiderhandlungen gegen sitzungsleitende Maßnahmen des Präsidenten einerseits und demonstrationsähnlichen Aktionen andererseits. ${ }^{91}$ Schließlich sollen als Bemessungszeitraum grundsätzlich drei Sitzungswochen gelten. ${ }^{92}$ Diese Zeitvorgabe ist jedoch weder gesetzlich vorgeschrieben noch zwingend. Der Präsident ist deshalb rechtlich nicht daran gehindert, in begründeten Fällen, auch dann einen Wiederholungsfall anzunehmen, wenn zwischen den ähnlich gelagerten Störungen ein größerer zeitlicher Abstand besteht. $^{93}$

Die Feststellung, dass ein Wiederholungsfall vorliegt, muss nicht ad-hoc erfolgen, da dies in bestimmten Fällen (zum Beispiel bei einer Störung durch eine Vielzahl nicht eindeutig identifizierbarer Mitglieder) schlicht nicht möglich ist. Vielmehr ist auch hier dem Präsidenten die Möglichkeit einzuräumen - analog zur Situation eines nachträglichen Ordnungsgeldes oder Ausschlusses -, den Sachverhalt umfassend aufzuklären und den Vorfall gründlich zu bewerten. ${ }^{94}$ Die Entscheidung über das Vorliegen eines Wiederholungsfalls müsste danach spätestens in der auf die Verletzung der Ordnung folgenden Sitzung des Bundestages im Plenum bekannt gegeben werden.

90 BT-Drucksache 17/5471 vom 12. April 2011, S. 4; BT-Drucksache 17/6309 vom 29. Juni 2011, S. 6; Heinrich G. Ritzel / Joseph Bücker / Hermann J. Schreiner, a.a.O. (Fn. 5), \$37, Anmerkung 4a).

91 Vgl. Heinrich G. Ritzel / Joseph Bücker / Hermann J. Schreiner, a.a.O. (Fn. 5), \$37, Anmerkung $4 a)$.

92 BT-Drucksache 17/5471 vom 12. April 2011, S. 4; BT-Drucksache 17/6309 vom 29. Juni 2011, S. 6.

93 Vgl. Heinrich G. Ritzel / Joseph Bücker / Hermann J. Schreiner, a.a.O. (Fn. 5), \$37, Anmerkung $4 a)$.

94 BT-Drucksache 17/6309 vom 29. Juni 2011, S. 7; Heinrich G. Ritzel / Joseph Bücker / Hermann J. Schreiner, a.a.O. (Fn. 5), \$37, Anmerkung 4b). 


\subsection{Sonderfall: nachträgliche Festsetzung eines Ordnungsgeldes}

Der Präsident hat die Möglichkeit, ein Ordnungsgeld auch nachträglich festzusetzen. Dies folgt aus $\$ 37$ Satz 3 GO-BT, der die Regelung über den nachträglichen Ausschluss $(\$ 38$ Abs. 2 GO-BT) für entsprechend anwendbar erklärt. Voraussetzung hierfür ist, dass sich der Präsident zum Zeitpunkt der Störung die Verhängung einer nachträglichen Sanktion ausdrücklich vorbehält. Er muss sich hinsichtlich der Sanktionsart (Ordnungsgeld oder Ausschluss) jedoch nicht sofort festlegen oder die Verletzung bereits als „gröblich“ beziehungsweise als „nicht nur geringfügig“ bezeichnen. ${ }^{95}$ Während der Sitzung reicht es aus, dass der Präsident „eine Verletzung der Ordnung“ feststellt, denn die Möglichkeit, nachträglich auch einen Sitzungsausschluss beziehungsweise ein Ordnungsgeld festsetzen zu können, soll den Präsidenten in die Lage versetzen, selbst zu ermitteln oder in geeigneter Weise feststellen zu lassen, wer die Ordnungsverletzung begangen hat, ob der betreffende Abgeordnete bereits zuvor die parlamentarische Ordnung verletzt hat und von welcher Qualität die Ordnungsverletzung ist, um sodann seine Entscheidung zu treffen. ${ }^{96}$ Dies betrifft insbesondere Fälle, in denen der Präsident sich aufgrund der konkreten Umstände daran gehindert sieht, ad-hoc den Vorgang umfassend und abschließend geschäftsordnungsrechtlich zu bewerten (zum Beispiel wegen fehlender Möglichkeit einer Identifizierung, weil eine Vielzahl von Störern vorliegt, wegen Maskierungen, vorgehaltenen Transparenten etc.).

Die Entscheidung über einen nachträglichen Sitzungsausschluss beziehungsweise ein nachträgliches Ordnungsgeld muss dabei spätestens in der auf die Verletzung der Ordnung folgenden Sitzung des Bundestages ausgesprochen werden ( $\$ 38$ Abs. 2 Satz 1 GO-BT). Neben der Sachverhaltsaufklärung und rechtlichen Prüfung dient die Entscheidungsfrist auch dazu, den Störern die Gelegenheit zu bieten, die Entscheidung durch ihr weiteres Verhalten, zum Beispiel eine sofortige Beendigung der Störung oder durch eine Entschuldigung, zu beeinflussen. ${ }^{97}$

Beschränkt sich der Präsident im Zeitpunkt der Störung nicht darauf, einen Ordnungsverstoß festzustellen, sondern erteilt bereits einen formellen Ordnungsruf, so steht dies gleichwohl einer nachträglichen Festsetzung eines Ordnungsgeldes oder einem nachträglichen Ausschluss wegen desselben Sachverhalts nicht entgegen. Dies folgt aus $\$ 37$ Satz 3 GO-BT, der ohne Einschränkungen auf 38 Abs. 2 Satz 3 GO-BT verweist. ${ }^{98}$ Voraussetzung hierfür ist jedoch, dass sich der Präsident nach dem Ordnungsruf eine weitergehende Sanktion vorbehalten hat. ${ }^{99}$

95 BT-Drucksache 17/6309 vom 29. Juni 2011, S. 7; BT-Drucksache 16/13492 vom 18. Juni 2009, S. 5; Heinrich G. Ritzel / Joseph Bücker / Hermann J. Schreiner, a.a.O. (Fn. 5), \$38 Anmerkung 6 b).

96 BT-Drucksache 17/6309 vom 29. Juni 2011, S. 7; BT-Drucksache 16/13492 vom 18. Juni 2009, S. 5.

97 BT-Drucksache 16/13492 vom 18. Juni 2009, S. 5.

98 Vgl. Heinrich G. Ritzel / Joseph Bücker / Hermann J. Schreiner, a.a.O. (Fn. 5), \$37, Anmerkung $3 \mathrm{~d})$.

99 Vgl. ebenda. 


\section{Sitzungsausschluss nach \38 GO-BT}

Zur Klarstellung, dass ein Sitzungsausschluss auch nach Einführung des Ordnungsgeldes weiterhin unter den bisherigen Voraussetzungen des $\$ 38$ GO-BT zulässig bleibt, ist dieser - quasi deklaratorisch - mit in das Abgeordnetengesetz (\$44a Abs. 5 Satz 3) aufgenommen worden. ${ }^{100}$ Der Gesetzgeber sah in Kenntnis der bisherigen parlamentarischen Praxis keinen Anlass, die Voraussetzungen für einen Ausschluss oder dessen Rechtsfolgen zu ändern oder gar einzuschränken. ${ }^{101}$ Auch sollte der Präsident in seinen ihm nach der Geschäftsordnung zustehenden Möglichkeiten, bei einem Sitzungsausschluss den jeweiligen Besonderheiten des Einzelfalles Rechnung tragen zu können, in keiner Weise beschränkt werden. ${ }^{102}$ Demgegenüber erfolgte die gesetzliche Verankerung des Ordnungsgeldes in $\$ 44 \mathrm{a}$ Abs. 5 AbgG, weil eine Änderung allein auf der Ebene der Geschäftsordnung mit Blick auf die vom Ordnungsgeld berührten Vermögensrechte der Abgeordneten als nicht ausreichend erachtet wurde. ${ }^{103}$

\subsection{Verfassungsrechtliche Einwände gegen den Sitzungsausschluss}

Der Sitzungsausschluss ist fester und tradierter Bestandteil der parlamentarischen Ordnungsgewalt ${ }^{104}$, nicht nur in deutschen Parlamenten. ${ }^{105}$ Auch in der Rechtsprechung ist die Möglichkeit eines Sitzungsausschlusses anerkannt, ohne dass hieran grundsätzliche verfassungsrechtliche Zweifel geäußert würden. ${ }^{106}$ In der Literatur mehren sich indes Stimmen, die den Sitzungsausschluss insbesondere wegen des mit ihm verbundenen Entzugs des Stimmrechts kritisch sehen beziehungsweise als verfassungswidrig einstufen. Dies gilt insbesondere für den über die laufende Sitzung hinausgehenden, so genannten weiteren Sitzungsausschluss. ${ }^{107}$

100 BT-Drucksache 17/5471 vom 12. April 2011, S.4.

101 BT-Drucksache 17/6309 vom 29. Juni 2011, S. 7; vgl. auch Redebeiträge der Abgeordneten Bernhard Kaster, Christian Lange und Thomas Strobl, BT-Plenarprotokoll 17/117 vom 30. Juni 2011, S. 13524 C, 13525 D, 13529 C.

102 Vgl. Heinrich G. Ritzel / Joseph Bücker / Hermann J. Schreiner, a.a.O. (Fn. 5), \$38, Anmerkung 2 c) nennt hier als Beispiel die ausnahmsweise Zulassung zu einer Abstimmung; vgl. auch BTPlenarprotokoll 17/25 vom 26. Februar 2010, S. 2198.

103 BT-Drucksache 17/5471 vom 12. April 2011, S. 3. Diese unterschiedliche Motivlage verkennen Albert Ingold / Sophie-Charlotte Lenski, a.a.O. (Fn. 8), S. 120, S. 121.

$104 \mathrm{Zu}$ den parlamentshistorischen Grundlagen Michael Köhler, a.a.O. (Fn. 9), S. 202 ff.; Heinrich G. Ritzel / Joseph Bücker / Hermann J. Schreiner, a.a.O. (Fn. 5), \$38, Anmerkung 1c).

105 Vgl. Art. 73 Geschäftsordnung Französische Nationalversammlung; Art. 60 Geschäftsordnung Twede Kamer (Niederlande). Auch im britischen Parlamentsrecht kennt man im Rahmen der Disciplinary and Penal Powers of the House das „Naming of a member and suspension“, http:// www.parliament.uk/documents/commons-information-office/g06.pdf (Abruf am 29. August 2012).

106 Das Bundesverfassungsgericht konstatierte 1959 in seinem so genannten Redezeiturteil (BVerfGE 10, S. 4, S. 13), dass die Ausübung der Redebefugnis des einzelnen Abgeordneten „den vom Parlament kraft seiner Autonomie gesetzten Schranken“ unterliege. Als Beispiel für solche Beschränkungen nennt das Gericht ausdrücklich den zeitweiligen Ausschluss eines Abgeordneten von der Teilnahme an Sitzungen. Vgl. auch zur entsprechenden landesrechtlichen Regelung in Sachsen VerfGH Sachsen, Urteil vom 3. Dezember 2010 - Vf. 77-I-10, juris Rz. 31.

107 Vgl. Klaus Abmeier, Die parlamentarischen Befugnisse der Abgeordneten des Deutschen Bundestages nach dem Grundgesetz, Berlin 1983, S. 240 ff.; Michael Härth, Der Ausschluss eines Abge- 
Die restriktivste Auffassung, die auch von Ingold und Lenski ${ }^{108}$ vertreten wird, geht davon aus, dass die Funktionsfähigkeit des Parlaments durch die Ausübung des Stimmrechts nicht beeinträchtigt wird, weil das Stimmrecht niemals ordnungswidrig ausgeübt oder mit Statusrechten anderer Abgeordneter in Konflikt geraten könne. Die Argumentation geht auf Martin Brandt und Dieter Gosewinkel zurück. ${ }^{109}$ Da dem Stimmrecht eine überragende Bedeutung für den Abgeordneten zukomme, erklären sie jeden Ausschluss für verfassungswidrig, der mit einem Entzug des Stimmrechts verbunden ist. Ein derart weitgehender Eingriff in den Kernbereich des Abgeordnetenstatus sei in noch höherem Maße als die Beschränkung des Anwesenheits- und Rederechts rechtfertigungsbedürftig. Eine Rechtfertigung lasse sich jedoch nicht finden, da das Stimmrecht keine Ordnungsstörung hervorrufen könne, es also an dem notwendigen unmittelbaren Bezug zum geahndeten Verhalten fehle. Wegen des unzulässigen Stimmrechtsentzugs müsse dem ausgeschlossenen Abgeordneten deshalb stets eine Teilnahme an der Abstimmung, die gegebenenfalls zu wiederholen sei, ermöglicht werden.

Dem ist entgegenzuhalten, dass mit dem Sitzungsausschluss kein Abstimmungsverhalten sanktioniert wird. Vielmehr ist der mit dem Ausschluss verbundene Entzug des Stimmrechts die (seltene) Konsequenz einer anderweitigen Verletzung der parlamentarischen Ordnung, die so gravierend ist, dass eine weitere Anwesenheit des Abgeordneten für alle Beteiligten untragbar wäre - ungeachtet der Frage, welcher Beratungsablauf einschließlich möglicher Abstimmungen noch ansteht. ${ }^{110}$ Es kommt demnach nicht darauf an, ob das Recht, in das eingegriffen wird, überhaupt imstande ist, eine Störung der Ordnung hervorzurufen. Eine derartige Korrelation ist dem parlamentarischen Ordnungsrecht fremd. Dies zeigt sich etwa in dem Fall, in dem ein Abgeordneter wegen Handgreiflichkeiten von der Sitzung ausgeschlossen würde. Ein Ausschluss hätte für den Abgeordneten neben dem Stimmrecht auch einen Verlust seines Rederechts zur Folge, obwohl er dieses ebenso wenig ordnungswidrig ausgeübt hat wie sein Stimmrecht.

Zudem geht schon die Prämisse fehl, der Abgeordnete könne sein Stimmrecht nicht ordnungswidrig ausüben. Abgesehen davon, dass bei einer Stimmabgabe per Handzeichen ( $\$ 48$ Abs. 1 GO-BT) durchaus ordnungswidrige Gesten denkbar sind, ist nicht allein der eigentliche Akt der Stimmabgabe entscheidend, sondern der Zeitraum während der parlamentarischen Beratung, in dem der Abgeordnete sein Stimmrecht ausüben kann. Die Beratung eines Tagesordnungspunktes im Plenum des Bundestages umfasst heute in der Praxis im Grundsatz drei Phasen: den Aufruf des Tagesordnungspunktes, die Aussprache (Debatte) und die Abstimmung. Wenn die dem Präsidenten vorliegende Rednerliste erschöpft ist, schließt er die Aussprache und leitet in die Abstimmungsphase über. Nach Eintritt in die Abstimmungs- beziehungsweise Wahlhandlung geht es ausschließlich um die Wahrnehmung des Stimmrechts. Die Sitzung kann zu diesem Zeitpunkt aber sehr wohl noch gestört oder

ordneten von der Teilnahme an Plenarsitzungen, in: ZRP, 17. Jg. (1984), S. 313, S. 316; Martin Brandt / Dieter Gosewinkel, a.a.O. (Fn. 25), S. 33, S. 37; Ulrich Franke, a.a.O. (Fn. 27), S. 110 ff.; Carmen Thiele, Regeln und Verfahren der Entscheidungsfindung innerhalb von Staaten und Staatenverbindungen, Berlin 2008, S. 429 ff.; Albert Ingold / Sophie-Charlotte Lenski, a.a.O. (Fn. 8), S. 120, S. 123.

108 Vgl. Albert Ingold / Sophie-Charlotte Lenski, a.a.O. (Fn. 8), S. 120, S. 123; ebenso Ulrich Franke, a.a.O. (Fn. 27), S. 110 ff.; Carmen Thiele, a.a.O. (Fn. 107), S. $429 \mathrm{ff}$.

109 Vgl. Martin Brandt / Dieter Gosewinkel, a.a.O. (Fn. 25), S. 33, S. 37.

110 Vgl. Harald Hemmer, Der Präsident des Landtags Nordrhein-Westfalen, Rheinbreitbach 2000, S. 313 (dort Fn. 361). 
gar die Abstimmung selbst be- oder verhindert werden (zum Beispiel Verhinderung von Stimmkarteneinwürfen, Entfernen der Wahlurne, Aufruf, nicht an der Abstimmung teilzunehmen etc.). ${ }^{111}$

Im Ergebnis argumentiert die Auffassung, die einem Ausschluss generell keine stimmrechtsentziehende Wirkung zukommen lassen möchte, zu sehr von einem ,individualrechtlichen" Standpunkt aus. Es wird ein Vorrang der Rede- und Abstimmungsfreiheit des Abgeordneten vor der Funktionsfähigkeit des Plenums postuliert. ${ }^{112}$ Das Bundesverfassungsgericht betont demgegenüber, dass bei der Bestimmung der verfassungsrechtlichen Rechte und Pflichten des Abgeordneten - der Notwendigkeit eines funktions- und repräsentationsfähigen Parlaments folgend - parlamentsrechtlich-funktionellen Gesichtspunkten prinzipiell der Vorrang gegenüber individualrechtlichen Gesichtspunkten einzuräumen ist. ${ }^{113}$ Wörtlich führt es in der sogenannten Nebeneinkünfte-Entscheidung aus: „Die Rechte des Abgeordneten richten sich nach den Erfordernissen demokratischer Repräsentation und stehen im Dienst der Erfüllung des Gemeinwohlauftrags des Deutschen Bundestages, nicht umgekehrt. Das freie Mandat ist zwar ein in der Gesellschaft verwurzeltes, aber innerhalb der Staatsorganisation wahrgenommenes Amt. "114

Nach dem Verständnis des Bundesverfassungsgerichts sind die durch Art. 38 Abs. 1 Satz 2 GG geschützten Statusrechte des Abgeordneten also in erster Linie Funktionsgarantien für den Bundestag als Volksvertretung. ${ }^{115}$ Es sind weniger Rechte als vielmehr Befugnisse beziehungsweise Kompetenzen, die funktional auf die organschaftliche Stellung des Abgeordneten bezogen und diese zu stützen bestimmt sind. ${ }^{116}$ Daraus folgt, dass die Statusrechte keinen Selbstzweck darstellen, sondern lediglich als Vehikel der parlamentarischen Willensbildung dienen. Diese Zweckbindung bedingt, dass das Rede- und Abstimmungsrecht des einzelnen Abgeordneten weniger schutzwürdig ist, wenn die parlamentarische Willensbildung beeinträchtigt wird. In diesen Fällen ist der Ausschluss zulässig, da ein Abgeordneter seine parlamentarischen Mitwirkungsrechte nicht dazu nutzen darf, das Parlament durch Störungen der Plenarsitzung daran zu hindern, seine staatsrechtlichen und politischen Aufgaben zu erfüllen. ${ }^{117}$

Erforderlich ist in jedem Einzelfall eine Abwägung zwischen den widerstreitenden Interessen der Sicherung der Aufgabenerfüllung des Parlaments einerseits und der Erfüllung eben dieser Funktion (Repräsentationsfunktion) durch Teilnahme aller Abgeordneten andererseits. Hierbei sind diverse Gesichtspunkte zu beachten: Art, Umfang Gewicht und Dauer der Störung, vorausgegangene vergleichbare Störungen, die gegebenenfalls in ihrer Häufung

111 Vgl. auch Art. 77, Geschäftsordnung Französische Nationalversammlung „versucht ein Abgeordneter im Plenum, die Beratungs- und Abstimmungsfreiheit zu lähmen (...)“.

112 Vgl. Harald Hemmer, a.a.O. (Fn. 110), S. 313.

113 BVerfGE 118, S. 277, S. 328.

114 Ebenda unter Hinweis auf BVerfGE 112, S. 118, S. 134.

115 Vgl. Martin Morlok, Parlamentarisches Geschäftsordnungsrecht zwischen Abgeordnetenrechten und politischer Praxis, in: JZ, Bd. 44 (1989), S. 11035 - 1047, S. 1035, S. 1037.

116 Hans Hugo Klein, a.a.O. (Fn. 23), Art. 138 Rz. 193; Hermann F. Schmid, a.a.O. (Fn. 14), S. 439, S. 528.

117 Vgl. Hans Hugo Klein, a.a.O. (Fn. 23), Art. 40 Rz. 103 (dort Fn. 9); Hans-Peter Schneider, a.a.O. (Fn. 30), Art. 38 Rz. 27 f.; Lars Brocker, a.a.O. (Fn. 11), Art. 40 Rz. 134; Hans Trossmann, a.a.O. (Fn. 11), $\$ 42$ Rz. 1ff.; Harald Hemmer, a.a.O. (Fn. 110), S. 312 f.; Michael Köhler, a.a.O. (Fn. 9), S. 206 ff.; Karl-Heinz Kleinschnittger, Die rechtliche Stellung des Bundestagspräsidenten, Düsseldorf 1963, S. $100 \mathrm{f}$. 
eine spezifische Gefahr für das Ansehen des Parlaments und die Autorität seiner Funktionsträger darstellen können sowie die Relevanz und Auswirkung eines Ausschlusses für das Abstimmungsergebnis. Ein Ausschluss dürfte danach etwa in Betracht kommen bei groben infamen Beleidigungen, hemmungslosem Lärmen, Handgreiflichkeiten oder nachhaltigen, wiederholten oder fortdauernden Störungen, indem zum Beispiel sitzungsleitenden Weisungen des Präsidenten konsequent zuwidergehandelt wird beziehungsweise an Äußerungen festgehalten wird, die bereits mit einem Ordnungsruf und/oder Ordnungsgeld bedacht worden sind. Gleiches könnte für Versuche gelten, eine Abstimmung zu stören oder einen Redebeitrag tatsächlich zu verhindern. ${ }^{118}$

Letztlich gilt es zu akzeptieren, dass dem Präsidenten ein ausreichendes Instrumentarium an Reaktionsmöglichkeiten zur Verfügung stehen muss. In einem solchen System stellt der Sitzungsausschluss das „letzte Mittel“ dar, um bewusstem, wiederholtem und sich eventuell steigerndem Fehlverhalten wirksam und nachhaltig begegnen zu können. In der jüngeren Praxis des Bundestages sind derartige Vorgänge zwar selten, das ordnungsrechtliche Sanktionssystem ist aber von Wahlperioden unabhängig, von früheren Erfahrungen geprägt und auch auf theoretisch denkbare zukünftige Entwicklungen hin ausgerichtet.

\subsection{Der weitere Sitzungsausschluss}

Zwischen einem Sitzungsausschluss für den Rest des Tages und für weitere Tage besteht in erster Linie ein zeitlicher und kein qualitativer Unterschied. Wenn man die Zulässigkeit des Sitzungsausschlusses prinzipiell bejaht, ist eigentlich kein Grund dafür ersichtlich, warum der Ausschluss nur unter einer engen zeitlichen Begrenzung erfolgen darf. ${ }^{119}$ Ein kurzer Sitzungsausschluss beseitigt zwar die Störung selbst, angesichts der Schwere der Ordnungsverletzung, die einem Sitzungsausschluss als „ultima ratio“ vorausgehen muss, kann der Sanktionscharakter und die Notwendigkeit einer nachhaltigen Reaktion indes nicht gänzlich unberücksichtigt bleiben, zumal die Geschäftsordnung explizit einen weiten Sanktionsrahmen vorsieht („,bis zu 30 Sitzungstagen“).

Die Frage, ob ein Abgeordneter über den laufenden Sitzungstag hinaus von Plenar- und Ausschusssitzungen ausgeschlossen werden kann, ist deshalb im Ergebnis davon abhängig, ob dem Parlament kraft Verfassung die Befugnis zusteht, mit der Ordnungsgewalt auch repressive und nicht lediglich präventive Zwecke zu verfolgen. Ist dies zulässig, kann die Beschränkung der Dauer des Ausschlusses auf die laufende Sitzung nicht mehr zwingend begründet werden. ${ }^{120}$

Dass mit der Ordnungsgewalt repressive Zwecke verfolgt werden können, wird zu Recht aus dem systematischen Zusammenhang abgeleitet, der zwischen der in der Geschäftsord-

118 Vgl. Heinrich G. Ritzel/ Joseph Bücker / Hermann J. Schreiner, a.a.O. (Fn. 5), § 38 Anmerkung 2 a); Lars Brocker, a.a.O. (Fn. 11), Art. 40 Rz. 134.

119 Vgl. Heinrich von Brentano, a.a.O. (Fn. 36), S. 59. Auch in der landesverfassungsgerichtlichen Rechtsprechung wird die Zulässigkeit einer zeitlichen Erstreckung des Sitzungsausschlusses über den laufenden Sitzungstag hinaus nicht bezweifelt; vgl. VerfGH Sachsen, Urteil vom 3. Dezember 2010 - Vf. 77-I-10, juris Rz. 31.

120 Vgl. Martin Brandt / Dieter Gosewinkel, a.a.O. (Fn. 25), S. 33, S. 36; anderer Auffassung Michael Härth, in: ZRP 1984, S. 313, S. 316, der einen Ausschluss für die laufende, allenfalls für die anschließende Sitzung für zulässig hält; Klaus Abmeier, a.a.O. (Fn. 107), S. 240 ff.; Ulrich Franke, a.a.O. (Fn. 27), S. $112 \mathrm{ff}$. 
nungsautonomie in Art. 40 Abs. 1 Satz 2 GG wurzelnden Ordnungsgewalt und dem Indemnitätsschutz aus Art. 46 Abs. 1 GG besteht. ${ }^{121}$ Die Indemnität schützt den einzelnen Abgeordneten bis zur Grenze der verleumderischen Beleidigung davor, für Abstimmungen oder Äußerungen, die er im Plenum oder in den Ausschüssen getan hat, außerhalb des Parlaments zur Verantwortung gezogen zu werden. Da der Abgeordnete somit nur hinsichtlich von Sanktionen außerhalb des Bundestages privilegiert wird, kann er im Umkehrschluss innerhalb des Parlaments kraft der durch Art. 40 Abs. 1 Satz 2 GG gewährleisteten Geschäftsordnungsautonomie „zur Verantwortung gezogen“ werden. Schneider bezeichnet die parlamentarischen Ordnungsmaßnahmen deshalb zutreffend auch als „funktionsspezifische Substitute des staatlichen Verfolgungsverbots zur Aufrechterhaltung der innerparlamentarischen Ordnung“. Folglich dürfen mit ihnen auch repressive Zwecke verfolgt werden. Wenn ein solches, zur Aufrechterhaltung der parlamentarischen Ordnung vorhandenes Sanktionssystem wie die $\$ \$ 36$ ff. GO-BT für besonders schwere Fälle („gröbliche Verletzung“) einen längerfristigen Ausschluss vorsieht, ist dies jedenfalls dann nicht zu beanstanden, wenn im Einzelfall der Ausschluss angemessen ist und die Dauer des Ausschlusses faktisch nicht einem Mandatsentzug gleichkommt. ${ }^{122}$

In Teilen der Literatur wird die Möglichkeit eines langfristigen Sitzungsausschlusses generell mit der Begründung abgelehnt beziehungsweise kritisch gesehen, dass bei knappen Mehrheitsverhältnissen, insbesondere dann, wenn die Stimme eines einzelnen Abgeordneten den Ausschlag gibt, die Funktionsunfähigkeit des Parlaments drohe („Pattsituation“). ${ }^{123}$ Es wird zudem die Gefahr gesehen, dass der Präsident sich bei einem Ausschluss dem Vorwurf der Manipulation ausgesetzt sehen könnte beziehungsweise bei einem Fehlgebrauch des Instruments Stimmverhältnisse gar gezielt manipulierbar sind. ${ }^{124}$ Deshalb sprechen sich einige Stimmen in der Literatur dafür aus, zu den Regelungen der GO/RT des Norddeutschen Bundes und der GO der Nationalversammlung vom 6. Februar 1919 zurückzukehren. ${ }^{125}$ Diese ließen einen Ausschluss nur für die laufende Sitzung zu. Zudem wurden während des Ausschlusses vorgenommene Abstimmungen über andere als Geschäftsordnungsfragen wiederholt, falls der Stimme des ausgeschlossenen Abgeordneten ausschlaggebende Bedeutung hätte zukommen können. Die Kritik ist im politischen Raum im Zusammenhang mit der Neufassung der $\$ \$ 36$ ff. GO-BT von der Fraktion Bündnis 90/ Die Grünen aufgegriffen worden. Der Antrag, die Möglichkeit eines längerfristigen Sitzungsausschlusses zu streichen, fand indes keine Mehrheit. ${ }^{126}$

121 Vgl. Michael Köhler, a.a.O. (Fn. 9), S. 179; Joseph Bücker, a.a.O. (Fn. 6), \$34 Rz. 11; Hans-Peter Schneider, a.a.O. (Fn. 30), Art. 46 Rz. 8; Martin Brandt / Dieter Gosewinkel, a.a.O. (Fn. 25), S. 33, S. 36; vgl. auch LVerfG Mecklenburg-Vorpommern, in: NordÖR 2009, S. 205, S. 207.

122 Vgl. Martin Brandt / Dieter Gosewinkel, a.a.O. (Fn. 25), S. 33, S. 36.

123 Vgl. Horst Wuttke, in: Heinz-Werner Arens, Geschäftsordnung des Schleswig-Holsteinischen Landtags, Kiel 1999, $\$ 68$ Rz. 7; Michael Köhler, a.a.O. (Fn. 9), S. 214; in der Vorauflage auch Heinrich G. Ritzel / Joseph Bücker / Hermann J. Schreiner, a.a.O. (Fn. 5), Vorbemerkung 4 a) zu $\$ \$ 36$ - 41; Hans Trossmann, a.a.O. (Fn. 11), \$42 Rz. 12.

124 BT-Drucksache 17/6353 vom 29. Juni 2011.

125 Vgl. Michael Köhler, a.a.O. (Fn. 9), S. 214; Heinrich G. Ritzel / Joseph Bücker / Hermann J. Schreiner, a.a.O. (Fn. 5), in der Vorauflage, Vorbemerkung 4 a) zu $\$ \$ 36-41$.

126 BT-Drucksache 17/6353 vom 29. Juni 2011; BT-Plenarprotokoll 17/117 vom 30. Juni 2011, S. 13528 C, S. 13531 B. 
Ohne die beschriebenen Gefahren gänzlich in Abrede stellen zu wollen, geht es jedoch zu weit, einen langfristigen Sitzungsausschluss generell zu verbieten. Dem Präsidenten würde hierdurch ohne Ansehen der besonderen Umstände des Einzelfalles von vornherein ein Instrument für Fälle aus der Hand genommen, die nach Abwägung der oben genannten Gesichtspunkte (Art, Intensität und Dauer der Störung) eine über den Ausschluss von der laufenden Sitzung hinausgehende Sanktion im konkreten Fall durchaus gerechtfertigt erscheinen lassen. ${ }^{127}$ Überzeugender ist es deshalb, den bei engen Mehrheitsverhältnissen beschriebenen Gefahren im Einzelfall dadurch zu begegnen, dass in Abweichung von der Geschäftsordnung ( $\$ 126$ GO-BT) ausgeschlossene Abgeordnete ausnahmsweise zu einer bedeutenden oder voraussichtlich knappen Abstimmung zugelassen werden. Eine besondere verfassungsrechtliche Bedeutung kommt demnach nur der Bemessung der Dauer des weiteren Ausschlusses zu. ${ }^{128}$ Hierfür lassen sich nur schwerlich nähere Hinweise und Richtlinien finden.

\section{Ordnungsgeld und Sitzungsausschluss: verfassungsrechtlich unbedenklich}

Die Einführung des Ordnungsgeldes stellt eine sinnvolle Erweiterung des Instrumentariums dar, das dem Präsidenten im Rahmen seiner Ordnungsgewalt zur Verfügung steht. Er wird hierdurch in die Lage versetzt, differenzierter und effizienter gegen die unterschiedlichsten Arten von Ordnungsstörungen vorzugehen. Darüber hinaus können durch das Ordnungsgeld öffentlichkeitswirksame Konfrontationen, wie zum Beispiel bei einer zwangsweisen Entfernung aus dem Sitzungssaal, vermieden werden. Die hiergegen vorgebrachten verfassungsrechtlichen Einwände vermögen ebenso wenig zu überzeugen wie die am Sitzungsausschluss geübte Kritik.

127 So hat der Sächsische Verfassungsgerichtshof zu Recht einen geschäftsordnungsrechtlich höchstmöglichen zehntägigen Sitzungsausschluss im Anschluss an zwei Ordnungsrufe, Weitersprechen trotz Wortentziehung und anschließendem Ausschluss und Nichtverlassen des Plenarsaals trotz mehrfacher Aufforderung nicht beanstandet (VerfGH Sachsen, Urteil vom 3. Dezember 2010 - Vf. 77-I-10, juris); vgl. auch VerfGH Sachsen, Beschluss vom 22. Juni 2012 - Vf. 58-I-12 zu dem bereits mehrfach erwähnten dreitägigen Sitzungsausschluss wegen des Nichtablegens von Kleidung der Marke „Thor Steinar“.

128 Vgl. Heinrich G. Ritzel/ Joseph Bücker / Hermann J. Schreiner, a.a.O. (Fn. 5), \$38, Anmerkung $5 \mathrm{~b})$. 